\title{
A Method for Incorporating Three-Dimensional Residual Stretches/Stresses into Patient-Specific Finite Element Simulations of Arteries
}

\author{
David M. Pierce ${ }^{\dagger}$, Thomas E. Fastl ${ }^{\ddagger}$, Borja Rodriguez-Vila ${ }^{\llbracket}$, \\ Peter Verbrugghe $^{\S}$, Inge Fourneau ${ }^{\S}$, Geert Maleux ${ }^{\S}$, \\ Paul Herijgers ${ }^{\S}$, Enrique J. Gomez $₫$, Gerhard A. Holzapfel ** \\ ${ }^{\dagger}$ Departments of Mechanical Engineering/Biomedical Engineering/Mathematics \\ University of Connecticut, 191 Auditorium Road, Unit 3139, Storrs, CT, USA 06269-3139 \\ $\ddagger$ Institute of Biomechanics, Graz University of Technology \\ Kronesgasse 5-I, 8010 Graz, Austria \\ ๑Bioengineering and Telemedicine Centre, Technical University of Madrid \\ Ciudad Universitaria s/n, 28040 Madrid, Spain \\ $\S$ Laboratory of Experimental Cardiac Surgery, Department of Cardiovascular Diseases \\ Gasthuisberg University Hospital, University of Leuven, 49 Herestraat, 3000 Leuven, Belgium
}

To appear in the J. Mech. Behav. Biomed. Mater.

March 25, 2015

${ }^{*}$ Corresponding author: holzapfel@ tugraz.at 


\section{Abstract}

The existence of residual stresses in human arteries has long been shown experimentally. Researchers have also demonstrated that residual stresses have a significant effect on the distribution of physiological stresses within arterial tissues, and hence on their development, e.g., stress-modulated remodeling. Through progress in medical imaging, image analysis and finite element (FE) meshing tools it is now possible to construct in vivo patient-specific geometries and thus to study specific, clinically-relevant problems in arterial mechanics via FE simulations. Classical continuum mechanics and FE methods assume that constitutive models and the corresponding simulations start from unloaded, stress-free reference configurations while the boundary-value problem of interest represents a loaded geometry and includes residual stresses. We present a pragmatic methodology to simultaneously account for both (i) the three-dimensional (3-D) residual stress distributions in the arterial tissue layers, and (ii) the equilibrium of the in vivo patient-specific geometry with the known boundary conditions. We base our methodology on analytically-determined residual stress distributions (J. R. Soc. Interface 7:787-799, 2010) and calibrate it using data on residual deformations (Ann. Biomed. Eng. 35:530-545, 2007). We demonstrate our methodology on three patient-specific FE simulations calibrated using experimental data. All data employed here are generated from human tissues - both the aorta and thrombus, and their respective layers - including the geometries determined from magnetic resonance images, and material properties and 3-D residual stretches determined from mechanical experiments. We study the effect of 3-D residual stresses on the distribution of physiological stresses in the aortic layers (intima, media, adventitia) and the layers of the intraluminal thrombus (luminal, medial, abluminal) by comparing three types of FE simulations: (i) conventional calculations; (ii) calculations accounting only for prestresses; (iii) calculations including both 3-D residual stresses and prestresses. Our results show that including residual stresses in patient-specific simulations of arterial tissues significantly impacts both the global (organ-level) deformations and the stress distributions within the arterial tissue (and its layers). Our method produces circumferential Cauchy stress distributions that are more uniform through the tissue thickness (i.e., smaller stress gradients in the local radial directions) compared to both the con- 
ventional and prestressing calculations. Such methods combined with appropriate experimental data, aim at increasing the accuracy of classical FE analyses for patient-specific studies in computational biomechanics and may lead to increased clinical application of simulation tools.

\section{Introduction}

Fung [1] and Vaishnav and Vossoughi [2] independently confirmed the existence of residual stresses in arteries. These residual stresses tend to homogenize the stress distribution within each arterial layer in the physiological state [3-5]. Numerous studies have shown that the inclusion of residual stresses in analyses of arteries under physiological loading conditions substantially reduces the variation in circumferential and axial stresses within the arterial wall [6-12]. Since residual stresses significantly affect the total stress state in the tissue, they also have significant impact on the in vivo state and development of arterial tissues. Examples include stress-modulated remodeling of arteries in health and disease (e.g., atherosclerosis, hypertension) [13-18].

Through progress in medical imaging, image analysis and finite element (FE) meshing tools it is now possible to extract patient-specific geometries from medical images of aortas and abdominal aortic aneurysms (AAAs), and thus to study specific, clinically-relevant problems in arterial mechanics via FE simulations. Such simulations allow additional insight into both human physiology and pathophysiology. The final aim of such simulations is to provide improved analysis tools and patient-specific information to help clinicians diagnose and treat arterial pathologies. Medical imaging is performed in vivo, and hence the reconstructed model geometry in the problem of interest represents the in vivo state, e.g., the aorta and AAA at physiological blood pressure. However, classical continuum mechanics and FE methods assume that constitutive models and the corresponding simulations start from an unloaded, stress-free reference configuration.

Two problems exist when applying such conventional approaches to patient-specific simulations of arteries: (i) the in vivo determined 'initial' geometry is not an unloaded reference configuration; (ii) the unloaded tissue itself is residually stressed. Computational methods of prestressing 
the FE model overcome the first problem so that, e.g., the initial (image derived) geometry is in equilibrium with the known physiological loads, cf., Gee et al. [19] and Weisbecker et al. [20]. The second problem, that of in vivo residual stresses in patient-specific simulations, has still not been thoroughly addressed in the biomechanics literature, hence the motivation for our current study.

There have been many theoretical studies investigating the causes and effects of residual stresses in arteries, cf., e.g., Bustamante and Holzapfel [21], Holzapfel and Ogden [22], Ren [23], Schröder and Brinkhues [24], Waffenschmidt and Menzel [25], but such works are outside our current focus on patient-specific FE-based simulations. Alastrué et al. [26] introduced a computational method to account for residual stresses in patient-specific simulations of arteries based on a single so-called 'opening angle' determined from a classical residual stress experiment (cf., e.g., Fung [1]). They applied a composition of deformation gradients to account for homogeneous deformation of an arterial ring from an opened (assumed as stress free) to a closed (residually stressed but unloaded) configuration that differs (hopefully little!) from the in vivo patientspecific geometry due to non-compatibility issues. They exercised their method on a slice of a human coronary artery and a 3-D human iliac artery obtained by segmenting computed tomography images. Computational models, residually stressed by this method, reproduce the opening angle experiment with reasonable accuracy. Furthermore, patient-specific simulations under in vivo conditions, i.e., applied internal pressure, demonstrate that the effect of residual stresses is significant, where transmural gradients change signs in the medial layer and stresses become nearly constant in the adventitia. In their analyses the authors did not account for the fact that the in vivo determined geometry is in equilibrium with physiological loads.

Later, Alastrué et al. [27] proposed an extended numerical framework for patient-specific modeling of vascular tissue in order to obtain a residually stressed and geometrically consistent model of the patient. They accounted for residual stresses via their previously published approach [26], while incorporating prestresses due to physiological loads by using an iterative approach based on a multiplicative decomposition of the deformation gradient. They demonstrated their framework by reconstructing a human carotid bifurcation from angiographic images, assuming a constant 
wall thickness for the artery and using a single set of material parameters experimentally determined from porcine tissue. Results show that incorporating residual stresses into patient-specific simulations dramatically affects the circumferential stresses by homogenizing the distribution and reducing gradients through the tissue thickness. Additionally, the deformed configurations of patient-specific geometries can differ significantly when comparing simulations with and without accounting for residual stresses.

Holzapfel et al. [28] investigated 3-D residual deformations for intact tissue strips of human aortas and for their separated layers in their passive states. From 11 abdominal aortas, the authors harvested 16 pairs of rings and axial strips, and cut the rings open. The intact tissue strips were allowed to relax for 16 hours before capturing images of the relaxed configurations. The strips were separated into their three layers and images were again recorded after another six hours of relaxation. The authors then used image processing and analysis to quantify residual stretches and curvatures. This study shows that residual deformations in arteries are three-dimensional (3-D) and thus cannot be described by a single parameter such as the opening angle. Their quantification and modeling therefore require consideration of stretching and bending, which are both highly layer-specific and axially dependent, and hence the methodology presented by, e.g., Alastrué et al. [26, 27], while a step forward, can not fully characterize the 3-D residual stress state in patient-specific simulations.

The purpose of this work is to present a methodology to simultaneously account for both (i) equilibrium of the in vivo patient-specific configuration with the known in vivo boundary conditions and (ii) the 3-D residual stress distributions known to exist in the arterial layers, and finally to study the importance of including 3-D residual stresses in patient-specific simulations of arteries. To this end, we propose a pragmatic approach to incorporate experimentally-determined residual stretches (stresses) into FE simulations of arterial tissues built from medical imaging data. We calculate a residual deformation gradient, for each Gauss quadrature point of the elements in each tissue layer, that reflects the kinematic transformation between a (nearly) stress-free, layer-separated reference configuration and the actual (possibly patient-specific) composite configuration. 
After validation analyses, we exercise the methodology, accounting for both residual stresses and prestresses, on complete patient-specific FE simulations of human AAA using state-of-theart anisotropic constitutive models (Gasser et al. [29] for the aortic tissue and Holzapfel et al. [30] for the intraluminal thrombus (ILT)), and corresponding experimentally determined material and structural parameters (Weisbecker et al. [31] and Tong et al. [32], respectively). Specifically, in order to investigate the effects of initial stresses (including both residual stresses and prestresses) on the stress distributions within the AAA wall and ILT, we perform and compare three different simulation methods: (i) conventional forward FE computations neglecting both prestresses and residual stresses; (ii) computations accounting only for prestresses using the Generalized Prestressing Algorithm (GPA) [20]; (iii) computations including both the 3-D residual stresses and the prestresses using our proposed methodology. As representative test cases we demonstrate results at two blood pressures (diastolic blood pressure of $85 \mathrm{mmHg}$ and systolic blood pressure of $135 \mathrm{mmHg}$ ) on three patient-specific geometries.

\section{Methods and Materials}

\subsection{Methodology to Determine Residual Stretches}

Herein we describe a methodology to determine the 3-D residual stretches (and thus stresses) in general patient-specific models of arteries that include the layered (intima, media, adventitia) structure of arterial walls. While the general methodology applies to any soft biological tissue where the appropriate data are available, we focus here on patient-specific modeling of the aorta based on the work of Holzapfel and Ogden [22] using data published on layer-specific residual deformations of human aortic tissue with non-atherosclerotic intimal thickening documented by Holzapfel et al. [28].

We define the deformation gradients mapping the 'nearly stress-free' reference configurations for each separated tissue layer (intima, media, adventitia) to the unloaded, patient-specific geometry $\mathbf{F}_{\mathrm{RS}}^{(i)}\left(r^{(i)}\right)$, with $i \in[\mathrm{I}, \mathrm{M}, \mathrm{A}]$ for the intima, media and adventitia, respectively, as (summarized 
from [22])

$$
\begin{aligned}
\mathbf{F}_{\mathrm{RS}}^{(\mathrm{I})}\left(r^{(\mathrm{I})}\right)= & \frac{L^{(\mathrm{I})}}{r^{(\mathrm{I})} k^{(\mathrm{I})} l}\left[A^{(\mathrm{I})^{2}}+k^{(\mathrm{I})} \frac{l}{L^{(\mathrm{I})}}\left(r^{(\mathrm{I})^{2}}-a^{(\mathrm{I})^{2}}\right)\right]^{(1 / 2)} \mathbf{e}_{r} \otimes \mathbf{E}_{R} \\
& +\frac{r^{(\mathrm{I})} k^{(\mathrm{I})}}{\left[A^{(\mathrm{I})^{2}}+k^{(\mathrm{I})} \frac{l}{L^{(\mathrm{I})}}\left(r^{(\mathrm{I})^{2}}-a^{(\mathrm{I})^{2}}\right)\right]^{(1 / 2)}} \mathbf{e}_{\theta} \otimes \mathbf{E}_{\Theta}+\frac{l}{L^{(\mathrm{I})}} \mathbf{e}_{z} \otimes \mathbf{E}_{Z}, \\
\mathbf{F}_{\mathrm{RS}}^{(\mathrm{M})}\left(r^{(\mathrm{M})}\right)= & \frac{L^{(\mathrm{M})} \pi}{r^{(\mathrm{M})} \beta l^{(\mathrm{M})} k^{(\mathrm{M})}}\left[A^{(\mathrm{M})^{2}}+\frac{\beta l^{(\mathrm{M})} k^{(\mathrm{M})}}{\pi L^{(\mathrm{M})}}\left(b^{(\mathrm{M})^{2}}-r^{(\mathrm{M})^{2}}\right)\right]^{(1 / 2)} \mathbf{e}_{r} \otimes \mathbf{E}_{R} \\
& +\frac{r^{(\mathrm{M})} \beta}{L^{(\mathrm{M})}} \mathbf{e}_{\theta} \otimes \mathbf{E}_{Z}+\frac{l^{(\mathrm{M})} k^{(\mathrm{M})}}{\pi\left[A^{(\mathrm{M})^{2}}+\frac{\beta l^{(\mathrm{M})} k^{(\mathrm{M})}}{\pi L^{(\mathrm{M})}}\left(b^{(\mathrm{M})^{2}}-r^{(\mathrm{M})^{2}}\right)\right](1 / 2)} \mathbf{e}_{z} \otimes \mathbf{E}_{\Theta},(2) \\
\mathbf{F}_{\mathrm{RS}}^{(\mathrm{A})}\left(r^{(\mathrm{A})}\right)= & \frac{L_{2}^{(\mathrm{A})} L_{3}^{(\mathrm{A})}}{\pi r^{(\mathrm{A})} l} \mathbf{e}_{r} \otimes \mathbf{E}_{X_{1}}+\frac{\pi r^{(\mathrm{A})}}{L_{2}^{(\mathrm{A})}} \mathbf{e}_{\theta} \otimes \mathbf{E}_{X_{2}}+\frac{l}{L_{3}^{(\mathrm{A})}} \mathbf{e}_{z} \otimes \mathbf{E}_{X_{3}},
\end{aligned}
$$

with $r^{(i)} \in\left[a^{(i)}, b^{(i)}\right]$, where $a^{(i)}$ and $b^{(i)}$ are the (deformed) internal and external radii of the layered tubes, respectively. For the human abdominal aorta, we specify values from the reference configuration $\left(A^{(I)}, B^{(I)}, L^{(\mathrm{I})}, k^{(\mathrm{I})}, A^{(M)}, B^{(M)}, L^{(\mathrm{M})}, k^{(\mathrm{M})}, L_{1}^{(\mathrm{A})}, L_{2}^{(\mathrm{A})}, L_{3}^{(\mathrm{A})}\right)$ and from the deformed configuration $\left(a^{(i)}, b^{(i)}, l\right)$, see Table 1 in [28]. The remaining parameter $\beta$ can be derived using traction continuity at the interfaces of the intima and media, as well as the media and adventitia [22]. After some manipulations we obtain

$$
\begin{aligned}
& \beta=\left[\frac{\mu^{(\mathrm{M})}\left(b^{(\mathrm{M})^{2}}-a^{(\mathrm{M})^{2}}\right)}{L^{(\mathrm{M})^{2}}}\right]^{-1 / 2} \\
& \times\left[\frac{\mu^{(\mathrm{I})} L^{(\mathrm{I})}\left(b^{(\mathrm{I})^{2}}-a^{(\mathrm{I})^{2}}\right)\left(A^{(\mathrm{I})^{2}} L^{(\mathrm{I})}-l a^{(\mathrm{I})^{2}} k^{(\mathrm{I})}\right)}{l^{2} a^{(\mathrm{I})^{2}} b^{(\mathrm{I})^{2}} k^{(\mathrm{I})^{2}}}\right. \\
& +\frac{2 \mu^{(\mathrm{I})} L^{(\mathrm{I})}\left(\log \left[\frac{b^{(\mathrm{I})}}{a^{(\mathrm{I})}}\right]-k^{(\mathrm{I})^{2}} \log \left[\sqrt{1+\frac{l k^{(\mathrm{I})}\left(b^{(\mathrm{I})^{2}}-a^{(\mathrm{I})^{2}}\right)}{L^{(\mathrm{I})} A^{(\mathrm{I})^{2}}}}\right]\right)}{l k^{(\mathrm{I})}} \\
& +\frac{\mu^{(\mathrm{M})}\left(b^{(\mathrm{M})^{2}}-a^{(\mathrm{M})^{2}}\right)^{2}\left(b^{(\mathrm{M})^{2}} B^{(\mathrm{M})^{2}}-a^{(\mathrm{M})^{2}} A^{(\mathrm{M})^{2}}\right)}{a^{(\mathrm{M})^{2}} b^{(\mathrm{M})^{2}}\left(B^{(\mathrm{M})^{2}}-A^{(\mathrm{M})^{2}}\right)^{2}}-\frac{2 \mu^{(\mathrm{M})}\left(b^{(\mathrm{M})^{2}}-a^{(\mathrm{M})^{2}}\right) \log \left[\frac{b^{(\mathrm{M})}}{a^{(\mathrm{M})}}\right]}{B^{(\mathrm{M})^{2}}-A^{(\mathrm{M})^{2}}}
\end{aligned}
$$

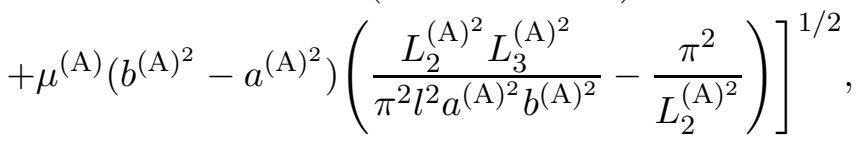

where $\mu^{(\mathrm{I})}, \mu^{(\mathrm{M})}$ and $\mu^{(\mathrm{A})}$ are stress-like material parameters, corresponding to the shear modulus of the underlying matrix materials (in the intima, media and adventitia respectively) in the 
Table 1: Determined geometrical parameters used to calculate the deformation gradients $\mathbf{F}_{\mathrm{RS}}^{(i)}\left(r^{(i)}\right)$, with $i \in[\mathrm{I}, \mathrm{M}, \mathrm{A}]$, mapping the 'nearly stress-free' reference configurations for each separated tissue layer (intima, media, adventitia) to the unloaded, patient-specific geometry; we also obtained $l=2.48 \mathrm{~mm}$. The interfaces between the layers $\left(b^{(\mathrm{I})}=a^{(\mathrm{M})}, b^{(\mathrm{M})}=a^{(\mathrm{A})}\right)$ were computed while all other values were determined experimentally (from Holzapfel et al. [28]).

\begin{tabular}{cll}
\hline Intima & \multicolumn{1}{c}{ Media } & \multicolumn{1}{c}{ Adventitia } \\
\hline \hline$A^{(\mathrm{I})}=7.50 \mathrm{~mm}$ & $A^{(\mathrm{M})}=8.41 \mathrm{~mm}$ & $L_{1}^{(\mathrm{A})}=0.205 \mathrm{~mm}$ \\
$B^{(\mathrm{I})}=7.76 \mathrm{~mm}$ & $B^{(\mathrm{M})}=8.99 \mathrm{~mm}$ & $L_{2}^{(\mathrm{A})}=18.3 \mathrm{~mm}$ \\
$L^{(\mathrm{I})}=2.58 \mathrm{~mm}$ & $L^{(\mathrm{M})}=2.52 \mathrm{~mm}$ & $L_{3}^{(\mathrm{A})}=2.29 \mathrm{~mm}$ \\
$k^{(\mathrm{I})}=1.19$ & $k^{(\mathrm{M})}=2.79$ & \\
$a^{(\mathrm{I})}=5.61 \mathrm{~mm}$ & $a^{(\mathrm{M})}=5.91 \mathrm{~mm}$ & $a^{(\mathrm{A})}=6.72 \mathrm{~mm}$ \\
$b^{(\mathrm{I})}=5.91 \mathrm{~mm}$ & $b^{(\mathrm{M})}=6.72 \mathrm{~mm}$ & $b^{(\mathrm{A})}=7.05 \mathrm{~mm}$ \\
\hline
\end{tabular}

reference configuration, which depend on experimentally determined material data (specified in Section 2.4.2). Once we have determined $\beta$, the parameter $l^{(\mathrm{M})}$ in (2) follows as

$$
l^{(\mathrm{M})}=\frac{\pi L^{(\mathrm{M})}}{\beta k^{(\mathrm{M})}} \frac{B^{(\mathrm{M})^{2}}-A^{(\mathrm{M})^{2}}}{b^{(\mathrm{M})^{2}}-a^{(\mathrm{M})^{2}}} .
$$

To evaluate the deformation gradients mapping the 'nearly stress-free' reference configurations for each separated tissue layer to the unloaded composite tissue, i.e. to determine the 3-D residual stretches, at the Gauss point locations within each tissue layer of a patient-specific FE model we map the analytically estimated residual stretches from (1)-(5) into an element-specific basis. For each Gauss quadrature point $j(j=1,2, \ldots, n$; where $n$ is the total number of Gauss points in the FE model) in each element within each tissue layer $i$, we define the local radial direction by a vector $\overline{\mathbf{n}}_{j}^{(i)}$ as follows. For each element in each tissue layer $i$ we determine the four nodes of the hexahedral element at the inner edge of the tissue layer, e.g., the surface of the media-adventitia interface for the adventitia, and fit a plane ${ }^{\mathrm{i}} \Gamma_{j}^{(i)}$ to these four nodal points using orthogonal distance regression. We define the unit normal vector to this inner plane as ${ }^{\mathrm{i}} \mathbf{n}_{j}^{(i)}$. For 
(a)

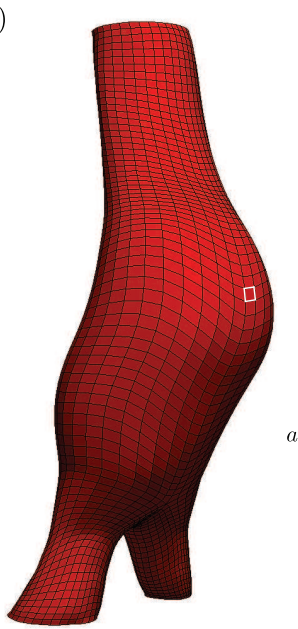

(b)

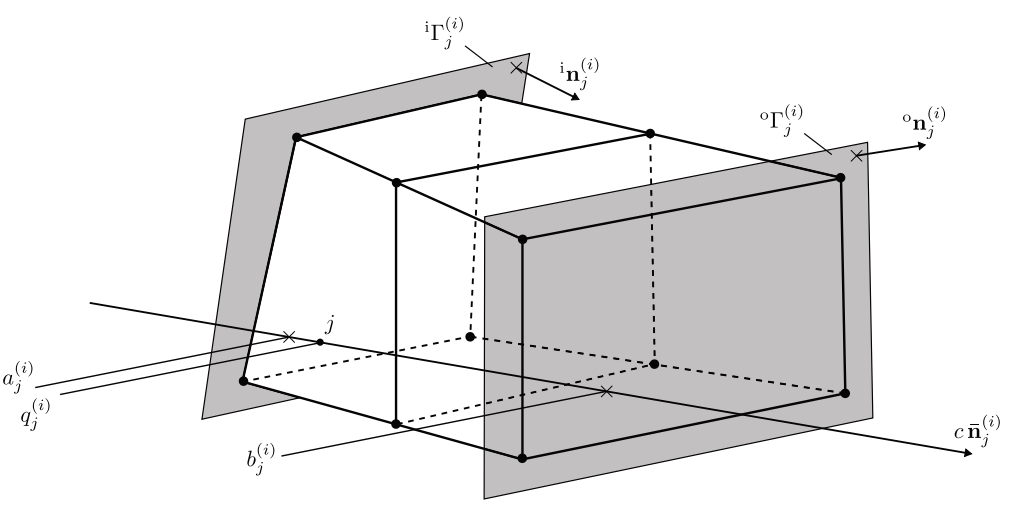

Figure 1: Representative schematic diagram of all the parameters we require to calculate the deformation gradients $\mathbf{F}_{\mathrm{RS}}^{(i)}\left(r^{(i)}\right)$, with $i \in[\mathrm{I}, \mathrm{M}, \mathrm{A}]$, at the Gauss point level: (a) patient-specific finite element mesh of the adventitia with selected elements, (b) perspective view of elements comprising the adventitia.

the same Gauss point $j$, we repeat this process by determining the four nodes of the hexahedral element at the outer surface of the tissue layer, e.g., the outer surface of the aorta for the adventitia (cf., Fig. 11), and we again fit a plane ${ }^{\circ} \Gamma_{j}^{(i)}$ to these four nodal points. We define the unit normal vector to this outer plane as ${ }^{\circ} \mathbf{n}_{j}^{(i)}$. Finally, we calculate the local radial direction by a vector as

$$
\overline{\mathbf{n}}_{j}^{(i)}=\frac{{ }^{\mathrm{i}} \mathbf{n}_{j}^{(i)}+{ }^{\circ} \mathbf{n}_{j}^{(i)}}{\left\|{ }^{\mathrm{i}} \mathbf{n}_{j}^{(i)}+{ }^{\circ} \mathbf{n}_{j}^{(i)}\right\|},
$$

where Einstein summation convention is not employed.

Next, we calculate a vector $c \overline{\mathbf{n}}_{j}^{(i)}$ (where $c \in \mathbb{R}^{+}$is an arbitrarily large, positive constant) defined to pass through Gauss point $j$, see Fig. 1 for a schematic representation. We then calculate three related distances (locations) from an arbitrary starting point along $c \overline{\mathbf{n}}_{j}^{(i)}: a_{j}^{(i)}$ is the distance to the intersection of $c \overline{\mathbf{n}}_{j}^{(i)}$ with the plane ${ }^{\mathrm{i}} \Gamma_{j}^{(i)}$ specified by unit normal ${ }^{\mathrm{i}} \mathbf{n}_{j}^{(i)} ; q_{j}^{(i)}$ is the distance to Gauss quadrature point $j$ along $c \overline{\mathbf{n}}_{j}^{(i)}$ and within tissue layer $i ; b_{j}^{(i)}$ is distance to the intersection of $c \overline{\mathbf{n}}_{j}^{(i)}$ with the plane ${ }^{\circ} \Gamma_{j}^{(i)}$ specified by unit normal ${ }^{\circ} \mathbf{n}_{j}^{(i)}$. Note that for a specific Gauss point $j$ within tissue layer $i, a_{j}^{(i)} \leq q_{j}^{(i)} \leq b_{j}^{(i)}$. 
We define a 'normalized' distance $f_{j}^{(i)}$ through the arterial tissue layer $i$ for Gauss point $j$ as

$$
f_{j}^{(i)}=\frac{q_{j}^{(i)}-a_{j}^{(i)}}{b_{j}^{(i)}-a_{j}^{(i)}},
$$

with $f_{j}^{(i)} \in[0,1]$. Finally, the 3-D residual stretches for Gauss point $j$ are determined using (1)-(5) and evaluated with

$$
r_{j}^{(i)}=a^{(i)}+f_{j}^{(i)}\left(b^{(i)}-a^{(i)}\right),
$$

where $a^{(i)}, b^{(i)}$ are from Table 1, We repeat this procedure for all Gauss quadrature points $j$ in all tissue layers $i$ of the FE model.

\subsection{Computational Algorithm: Sequential Residual Stressing and Prestressing}

Two problems exist when applying classical FE-based computational approaches to patientspecific simulations of arteries: (i) the problem of residual stresses (handled theoretically in Section 2.1); (ii) the in vivo determined 'initial' geometry is not an unloaded reference configuration. To address both problems, we apply the Generalized Prestressing Algorithm (GPA) [20].

Conventional FE codes generally start from an undeformed and stress-free reference configuration $\Omega_{0}$ and update the displacement $\mathbf{u}$ for each converged iteration step corresponding to time increment $\Delta t$ with the displacement increment $\Delta \mathbf{u}$, i.e., $\mathbf{u}_{t+1}=\mathbf{u}_{t}+\Delta \mathbf{u}$. The subscript $t+1$ (advancing the solution to $t+\Delta t$ ) denotes values from the current time step, whereas $t$ denotes values with respect to the previous time step. In the GPA the incremental update of the displacement is replaced by the incremental update of the deformation gradient. Specifically, we define the increment of the deformation gradient as [19, 20]

$$
\Delta \mathbf{F}=\mathbf{I}+\frac{\partial \Delta \mathbf{u}}{\partial \mathbf{x}_{t}}
$$

When storing $\mathbf{F}_{t}$ as a history variable, we update the deformation gradient [20]

$$
\mathbf{F}_{t+1}=\Delta \mathbf{F F}_{t}
$$

Hence, in this approach we calculate an incremental deformation gradient from the incremental displacement that results from each converged iteration step. We then delete the calculated 


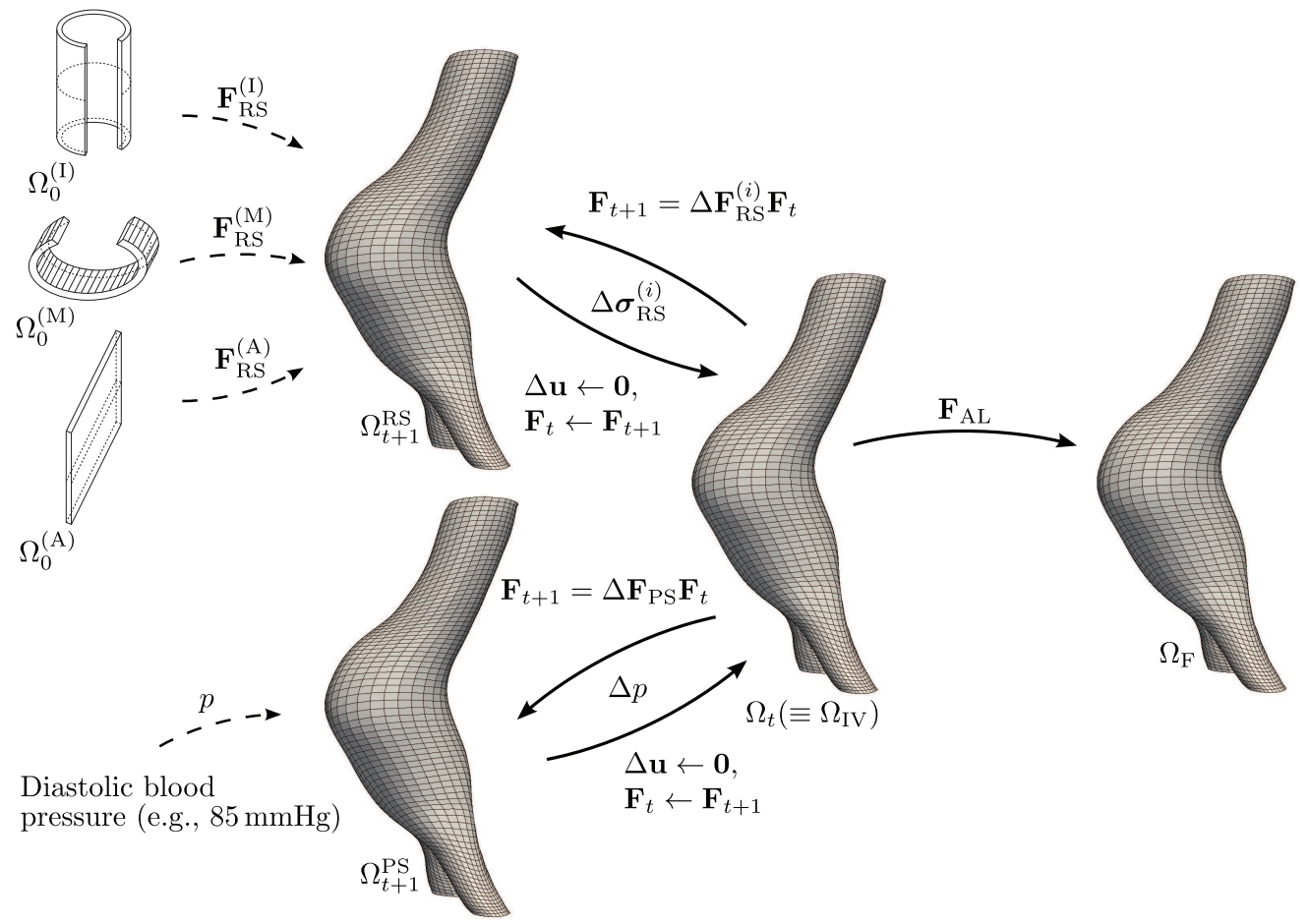

Figure 2: Methodology to sequentially include both residual stresses $\sigma_{\mathrm{RS}}^{(i)}(i \in[\mathrm{I}, \mathrm{M}, \mathrm{A}])$ and prestresses (resulting from, e.g., diastolic blood pressure $p=85 \mathrm{mmHg}$ ) into the in vivo, imaged configuration $\Omega_{\mathrm{IV}}$ of the finite element model and continue the simulation under an arbitrary load.

incremental displacement, returning us to the initial geometry, and update the total deformation gradient with the incremental deformation gradient. To do this, we define the total deformation gradient as an additional history variable during computation. Our algorithm thus builds up an effective total deformation gradient field without changing the initial geometry.

For the patient-specific AAA simulation, both residual stresses and prestresses must be incorporated to determine a state of equilibrium (at each Gauss point) in the initial, image-derived configuration. We use a sequential approach to accomplish this goal, first determining the distribution of residual stresses in the imaged, in vivo configuration and second accounting for the prestresses, again in the in vivo configuration. This approach is outlined schematically in Fig. 2 Following Fig. 2, we start our methodology at the imaged, in vivo geometry $\Omega_{\mathrm{IV}}$. Using the GPA, we iteratively apply increments $\Delta \mathbf{F}_{\mathrm{RS}}^{(i)}$ of the deformation gradients $\mathbf{F}_{\mathrm{RS}}^{(i)}\left(r^{(i)}\right)$, from (1)-(5), 
which deform $\Omega_{t} \equiv \Omega_{\mathrm{IV}}$ to configuration $\Omega_{\mathrm{t}+1}^{\mathrm{RS}}$. Once the solution converges, determining $\Omega_{\mathrm{t}+1}^{\mathrm{RS}}$, we delete the displacement $\Delta \mathbf{u} \leftarrow \mathbf{0}$ and update the history term for the deformation gradient $\mathbf{F}_{t} \leftarrow \mathbf{F}_{t+1}$. We repeat this iterative process (top, left-hand branch in Fig. (2) until the full layerspecific residual stresses $\boldsymbol{\sigma}_{\mathrm{RS}}^{(i)}$, resulting from the layer-specific deformations from the 'nearly' stress-free configurations $\Omega_{0}^{(i)}$ to the unloaded composite aorta configuration, are accounted for within the imaged, in vivo geometry $\Omega_{\mathrm{IV}}$.

Next, we use a similar process to account for the in vivo boundary conditions, e.g., the internal diastolic blood pressure $p=85 \mathrm{mmHg}$. Again using the GPA, we iteratively apply increment $\Delta \mathbf{F}_{\mathrm{PS}}$ of the deformation gradient resulting from the internal pressure increments $\Delta p$, which deform $\Omega_{t} \equiv \Omega_{\mathrm{IV}}$ to configuration $\Omega_{\mathrm{t}+1}^{\mathrm{PS}}$. Once the solution converges, determining $\Omega_{\mathrm{t}+1}^{\mathrm{PS}}$, we delete the displacement $\Delta \mathbf{u} \leftarrow \mathbf{0}$ and update the history term for the deformation gradient $\mathbf{F}_{t} \leftarrow$ $\mathbf{F}_{t+1}$. We repeat this iterative process (bottom, left-hand branch in Fig. 2) until the full in vivo boundary conditions are accounted for within the imaged, in vivo geometry $\Omega_{\mathrm{IV}}$.

Thus, our methodology concludes at the in vivo geometry that includes an internal stress distribution, accounting for both residual stresses and prestresses, in equilibrium with the known boundary conditions, e.g., diastolic blood pressure. From this point, we can apply arbitrary loads to study boundary-value problems of interest (right-hand branch in Fig. 2), resulting in the deformation gradient $\mathbf{F}_{\mathrm{AL}}$ which deforms $\Omega_{t}\left(\equiv \Omega_{\mathrm{IV}}\right)$ to configuration $\Omega_{\mathrm{F}}$. We accomplish the complete simulation methodology within the FE code FEAP (University of California at Berkeley, CA, United States) [33].

\subsection{Validation Analyses}

To validate our methodology for incorporating residual stresses into arterial tissues we start from the 'nearly' stress-free configurations given by Holzapfel et al. [28] as the layer-separated reference configurations determined experimentally. Therein, the authors investigate 3-D residual deformations for intact tissue strips of human aortas and for their separated layers in their passive states. 
We model 'nearly' stress-free reference configurations for each layer $\Omega_{0}^{(i)}(i \in[\mathrm{I}, \mathrm{M}, \mathrm{A}])$ by a segment with two curvatures representing the average stretch and curvature from 11 aortic tissue samples. Applying deformations to the FE models of these three individual layers we reconstruct the final cylindrical configuration from Holzapfel and Ogden [22].

In the final configuration we verify that the 3-D stress distributions predicted by the FE simulation match those predicted analytically, i.e., we reproduce the published analytical results. To reproduce these results we employ the material model and layer-specific parameters consistent with those used to produce the analytical results: the incompressible neo-Hookean model with parameters $\mu^{(\mathrm{I})}=39.8 \mathrm{kPa}, \mu^{(\mathrm{M})}=31.4 \mathrm{kPa}$ and $\mu^{(\mathrm{A})}=17.3 \mathrm{kPa}$, for the intima, media and adventitia respectively [22,34].

In addition to testing the method, the validation allows us to identify the influence of the approximation made by neglecting the minor curvature measured experimentally in the (nearly) stress-free configuration.

\subsection{Representative Patient-Specific Analyses}

\subsubsection{Patient-Specific Geometries and Meshes}

If there were no contraindications, we performed Magnetic Resonance Imaging (MRI) studies using a 1.5 T scanner (Aera; Siemens, Erlangen, Germany) and the protocol outlined in TarjueloGutiérrez et al. [35]* We collected three sets of patient-specific MR Images from patients with an AAA having a diameter of greater then $5 \mathrm{~cm}$. We segment and process the MRI data (axial slices) to generate our patient-specific geometries following the approach of Tarjuelo-Gutiérrez et al. [35]. Thus, we delineate three 3-D binary images: the outer arterial wall contour, the lumen contour and the thrombus contour (where ILT exists). To define the basic geometry we use the lumen contour as the inner wall boundary unless thrombus is present, in which case we take the thrombus contour as the inner wall. The thrombus contour defines the outer boundary of the ILT,

\footnotetext{
*The clinical trial center of the University Hospitals of Leuven registered the MRI study (study number S52774) and ethical approval was obtained from the ethical committee UZ Leuven.
} 
while the lumen contour defines its inner boundary. The outer arterial wall contour defines the outer boundary such that, in conjunction with the imaging planes at the extremes, the contours define volumes.

We base our FE mesh on direct meshing of the patient-specific geometries using almost exclusively hexahedral elements (we required some collapsed hexahedra [36] at the edge of the ILT), again following Tarjuelo-Gutiérrez et al. [35]. To begin, for completeness, we subdivide the volume of the AAA with the corresponding ILT into three individual vessels using three planes defined by five manually selected points: (i) Anterior - anterior cranial to the bifurcation on the lumen surface; (ii) Posterior - posterior cranial to the bifurcation on the lumen surface; (iii) Caudal - centered and caudal to the bifurcation on the wall surface; (iv) Dexter - dexter cranial to the bifurcation on the lumen surface; (v) Sinister - sinister cranial to the bifurcation on the lumen surface. The 3-D location of each point affects the shapes of the resulting elements and thus the quality of the meshes. We then carefully divide the three vessels into six sections in order to achieve suitable elements at the transition zones between independent sections. Next we generate three quadrilateral meshes (the nodes of which are subsequently used to construct the FE mesh with conforming hexahedral elements) defined by: (i) the outer contour of the AAA wall; (ii) the contour of the lumen; (iii) the inner contour of the AAA wall. We calculate a center line using the lumen surface and the quadrilateral mesh of the outer contour of the AAA wall as a reference. This mesh then serves as a reference to update the position of nodes defining the remaining quadrilateral meshes in order to generate radially aligned quadrilaterals at the outer and the inner boundaries of the AAA wall. Finally, we join these quadrilateral elements to form well-shaped hexahedral elements. An analogous process is repeated to generate hexahedral elements for a conforming mesh of the ILT. In locations where there is no ILT this method creates hexahedral elements with zero volume which we remove.

Finally, both the aortic wall (cf., [37]) and ILT (cf., [32]) constitute three layers. To model these features, we use the inner and outer edges of both the aortic wall and the ILT to reconstruct an accurate representation of the true three-layer structures. Starting from the two-layer mesh, i.e., the artery and thrombus, each hexahedra is divided into three hexahedra in the local radial direc- 
tion using empirically determined through-the-thickness ratios determined from human tissues: for the aortic wall - intima 0.14 , media 0.49 , and adventitia 0.37 [38]; and for the thrombus luminal 0.34 , medial 0.38 , and abluminal 0.28 [32]. To ensure solution accuracy, we refine the resulting six-layer mesh by further dividing existing hexahedra and testing both convergence of the solution (across mesh densities) and quality of the resulting hexahedral elements.

Among all the metrics available as a measure of initial mesh quality, the scaled Jacobian is most commonly used in FE simulations for solid structures [39]. We use the open source program ParaView [40], a multi-platform data analysis and visualization application including the Verdict library [41], to calculate and visualize the scaled Jacobian distribution of our elements. Therein, we evaluate the Jacobian, the determinant of the discrete Jacobian matrix, at the element center using the principal axes, and at each node of the hexahedral element as the triple scalar product (also called mixed or box product) of the edges connected to that node [35]. The scaled Jacobian, defined as the minimum value determined when normalizing each Jacobian against the length of its corresponding three vectors in the box product, takes the range [-1,1] for hexahedral elements where -1 indicates the worst possible elements and +1 the best. Solid analysis requires, at a minimum, a positive scaled Jacobian [39]. Generally, to ensure solution robustness, the scaled Jacobian should be greater than or equal to 0.5 [41]. We adjust our FE meshes until this quality metric indicates elements of high quality. Note we exclude collapsed hexahedra from this analysis since this quality metric is not applicable to such elements.

\subsubsection{Constitutive Models}

To model the response of the aortic wall and the thrombus determined from experiments, we use nonlinear, finite strain constitutive models based on convex strain-energy functions $\Psi$. We employ a multiplicative split of $\Psi$ into volumetric and isochoric contributions, using the isochoric deformation gradient $\overline{\mathbf{F}}=J^{-1 / 3} \mathbf{F}$, where $J$ is the determinant of the deformation gradient $\mathbf{F}$, and the corresponding isochoric right Cauchy-Green tensor is $\overline{\mathbf{C}}=J^{-2 / 3} \mathbf{C}$, where $\mathbf{C}=\mathbf{F}^{\mathrm{T}} \mathbf{F}$, see, e.g., [42]. We assume the decoupled form $\Psi=U(J)+\bar{\Psi}$, where the volumetric contribution is particularized as $U(J)=K(J-1)^{2} / 2$; here $K$ is a stress-like material parameter that, in the 
case of isochoric $(J=1)$ deformation, degenerates to a non-physical (positive) penalty parameter used to enforce incompressibility. We choose $\bar{\Psi}$ as

$$
\bar{\Psi}\left(\bar{I}_{1}, \bar{I}_{4}, \bar{I}_{6}\right)=\bar{\Psi}_{\mathrm{m}}\left(\bar{I}_{1}\right)+\bar{\Psi}_{\text {aniso }}^{k}\left(\bar{I}_{1}, \bar{I}_{4}, \bar{I}_{6}\right)
$$

where $\bar{I}_{1}=\operatorname{tr} \overline{\mathbf{C}}, \bar{I}_{4}=\overline{\mathbf{C}}: \mathbf{M} \otimes \mathbf{M}, \bar{I}_{6}=\overline{\mathbf{C}}: \mathbf{M}^{\prime} \otimes \mathbf{M}^{\prime}$ and $k=(\mathrm{a}, \mathrm{t})$ stands for artery and thrombus, respectively. The vectors $\mathbf{M}$ and $\mathbf{M}^{\prime}$ denote the principal directions of fiber reinforcement (e.g., the collagen fibers) in the reference configuration, with $|\mathbf{M}|=\left|\mathbf{M}^{\prime}\right|=1$.

For both the layers of arterial wall and ILT, we select the neo-Hookean model to represent the isotropic matrix material, and hence specify $\bar{\Psi}_{\mathrm{m}}$ as $\mu\left(\bar{I}_{1}-3\right) / 2$, where $\mu>0$ is a stress-like material parameter, corresponding to the shear modulus of the underlying matrix material in the reference configuration (specified as $\mu^{(i)}$, with $i \in[\mathrm{I}, \mathrm{M}, \mathrm{A}]$, for the intima, media and adventitia of the arteries in Section 2.1).

We capture the anisotropic, nonlinear response of the collagen fiber network in the layers of the aortic wall using [29,31]

$$
\bar{\Psi}_{\text {aniso }}^{\mathrm{a}}\left(\bar{I}_{1}, \bar{I}_{4}, \bar{I}_{6}\right)=\frac{k_{1}}{2 k_{2}} \sum_{l=4,6}\left\{\exp \left\{k_{2}\left[\kappa \bar{I}_{1}+(1-3 \kappa) \bar{I}_{l}-1\right]^{2}\right\}-1\right\},
$$

where the superscript a stands for artery, $k_{1}>0$ is a stress-like material parameter, $k_{2}>0$ is a dimensionless parameter, while $\kappa \in[0,1 / 3]$ is a dimensionless parameter representing dispersion of the collagen fiber orientations about the principal directions $\mathbf{M}$ and $\mathbf{M}^{\prime}$. We capture the anisotropic, nonlinear response of the ILT within the thrombus using [30, 32, 43]

$$
\bar{\Psi}_{\text {aniso }}^{\mathrm{t}}\left(\bar{I}_{1}, \bar{I}_{4}, \bar{I}_{6}\right)=\frac{k_{1}}{2 k_{2}} \sum_{l=4,6}\left\{\exp \left\{k_{2}\left[(1-\rho)\left(\bar{I}_{1}-3\right)^{2}+\rho\left(\bar{I}_{l}-1\right)^{2}\right]\right\}-1\right\},
$$

where the superscript $\mathrm{t}$ stands for thrombus and $\rho \in[0,1]$ is a dimensionless parameter representing dispersion about the principal direction of reinforcement in the ILT. Tension/compression nonlinearity in $\bar{\Psi}$ stems from conditional statements that both anisotropic terms $\left(\bar{\Psi}_{\text {aniso }}^{\mathrm{a}}\right.$ and $\bar{\Psi}_{\text {aniso }}^{\mathrm{t}}$ ) only contribute to the stored strain energy when $\bar{I}_{l}>1, l=4,6$, such that the stress response is only non-zero in tension. 
Table 2: Median material and structural parameters for the intima, media and adventitia of human abdominal aorta determined from nine tissue samples (mean age $61.0 \pm 11 \mathrm{yr}$ ) [31].

\begin{tabular}{rccccc}
\hline & $\mu[\mathrm{kPa}]$ & $k_{1}[\mathrm{kPa}]$ & $k_{2}[-]$ & $\varphi\left[^{\circ}\right]$ & $\kappa[-]$ \\
\hline \hline Intima & 44.0 & $10.1 \times 10^{3}$ & 0.00 & 40.5 & 0.25 \\
Media & 28.0 & $0.81 \times 10^{3}$ & 12.4 & 39.1 & 0.18 \\
Adventitia & 10.0 & $0.38 \times 10^{3}$ & 3.35 & 40.6 & 0.11 \\
\hline
\end{tabular}

Table 3: Mean material and structural parameters for the luminal, medial and abluminal layers of human intraluminal thrombus determined from 43 thrombi samples (mean age $67.0 \pm 6 \mathrm{yr}$ ) [32].

\begin{tabular}{rccccc}
\hline & $\mu[\mathrm{kPa}]$ & $k_{1}[\mathrm{kPa}]$ & $k_{2}[-]$ & $\varphi\left[^{\circ}\right]$ & $\rho[-]$ \\
\hline \hline Luminal & 19.4 & 15.9 & 2.7 & 84.1 & 0.33 \\
Medial & 14.2 & 6.0 & 0.07 & 86.7 & 0.05 \\
Abluminal & 10.2 & 2.9 & 0.03 & 89.1 & 0.05 \\
\hline
\end{tabular}

In both material models, the vectors $\mathbf{M}$ and $\mathbf{M}^{\prime}$ denote the directions of principal anisotropy in the reference configuration. We determine these directions, according to experimental data, symmetrically with respect to the local circumferential direction of the artery, and thus both directions are uniquely defined by the structural parameter $\varphi$, the angle between the circumferential direction of the artery and the fiber directions that lay in the plane of the tissue (i.e., a plane normal to the local radial direction). We implement these data element-wise into our finite element models using a custom Matlab (The MathWorks, Inc., Natick, MA) code, cf., Kiousis et al. [44].

Table 2 provides the median constitutive parameters $\mu, k_{1}, k_{2}, \varphi$ and $\kappa$ for the intima, media and the adventitia of the human abdominal aorta, as determined from nine tissue samples (mean age $61.0 \pm 11$ yr) from Weisbecker et al. [31]. Similarly, Table 3 provides the mean constitutive parameters $\mu, k_{1}, k_{2}, \varphi$ and $\rho$ for the three individual layers of ILT (luminal, medial and abluminal) determined from 43 samples of thrombi (mean age $67.0 \pm 6 \mathrm{yr}$ ) from Tong et al. [32]. 


\subsection{Representative Simulations}

In order to exercise our methodology and investigate the effect of initial stresses, including both residual stresses and prestresses, on the stress distributions within the AAA wall and the ILT we perform three different types of simulations: (i) conventional forward computations neglecting both residual stresses and prestresses, termed 'conventional' calculations; (ii) computations accounting only for prestresses using the GPA [20], termed 'prestressing' calculations; (iii) computations including both the 3-D residual stresses, according to (1)-(8), and prestresses according to our proposed algorithm (Section 2.2), termed 'novel' calculations. As representative test cases we demonstrate results on three patient-specific geometries. In these simulations we assume that the patients, suffering from AAAs, have a diastolic blood pressure (DBP) of $85 \mathrm{mmHg}$ and a systolic blood pressure (SBP) of $135 \mathrm{mmHg}$, classified as 'prehypertension' according to [45]. The medical images, i.e., MRI, are gated manner at DBP, important to note for pressurization using the GPA [20].

\section{Results}

\subsection{Validation Analyses}

We validate the accuracy of our computationally-based methodology by recreating analyticallydetermined 3-D residual stress distributions according to Holzapfel and Ogden [22] using detailed experimental data published on residual deformations from Holzapfel et al. [28]; Fig. 3 ] shows the comparison. Figures $3(a),(c),(e)$ show the normal components of the 3-D stress matrix versus the normalized radius for a three-layer arterial tissue composite in the unloaded, residually-stressed state, and the corresponding 3-D FE representations of the circumferential (b), the axial (d) and the radial (f) stress. Specifically, Fig. 3(c) shows the equilibrium (ensuring that the axial loads on the ends of the artery are zero) stress distribution $\left(\sigma_{z z}=\tilde{\sigma}_{z z}-\bar{\sigma}_{z z}\right.$, where $\tilde{\sigma}_{z z}$ are the FE simulated stresses and $\bar{\sigma}_{z z}$ is the mean axial stress), consistent with the definition from Holzapfel and Ogden [22]. Figure 3] d) shows the corresponding simulated stress $\tilde{\sigma}_{z z}$ in the 


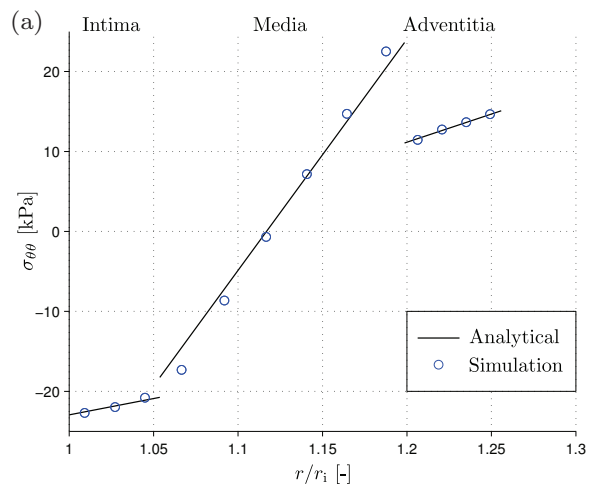

(b)
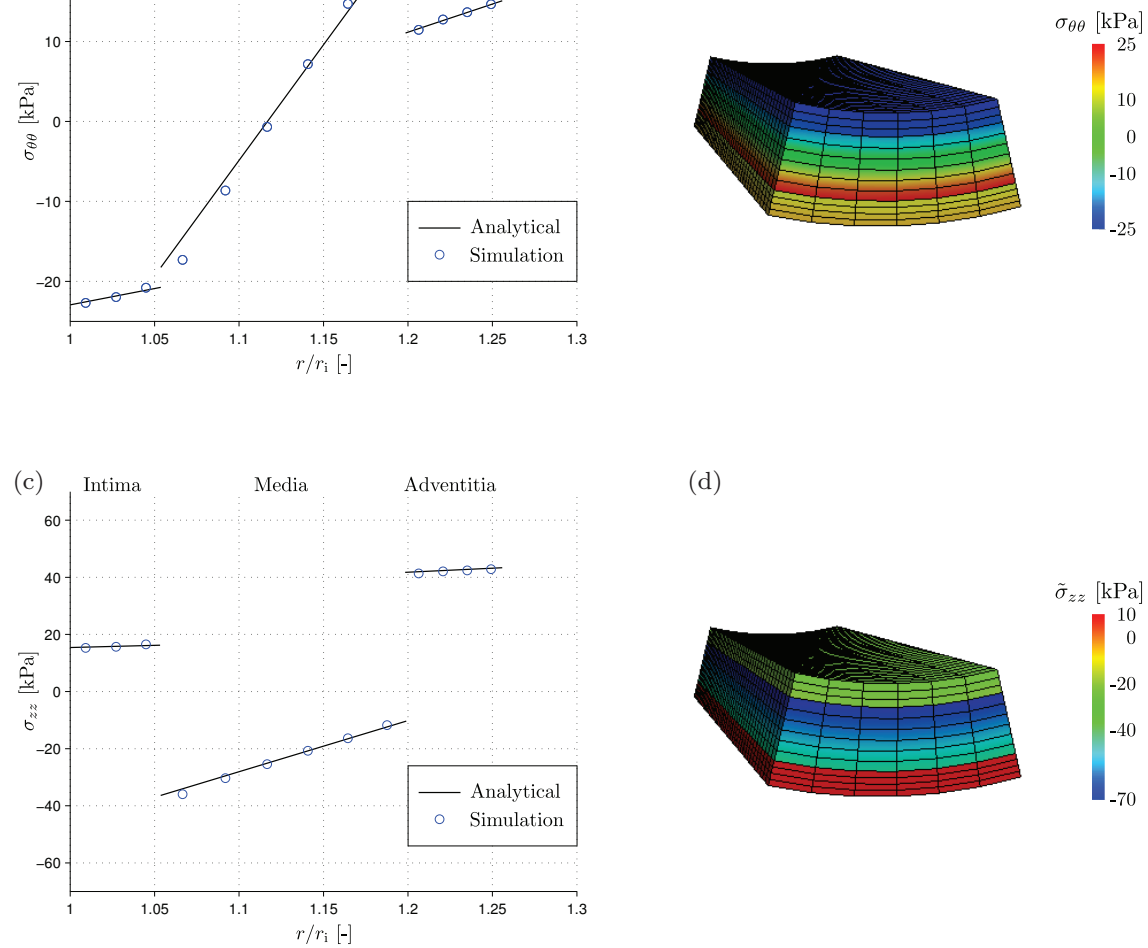

(d)
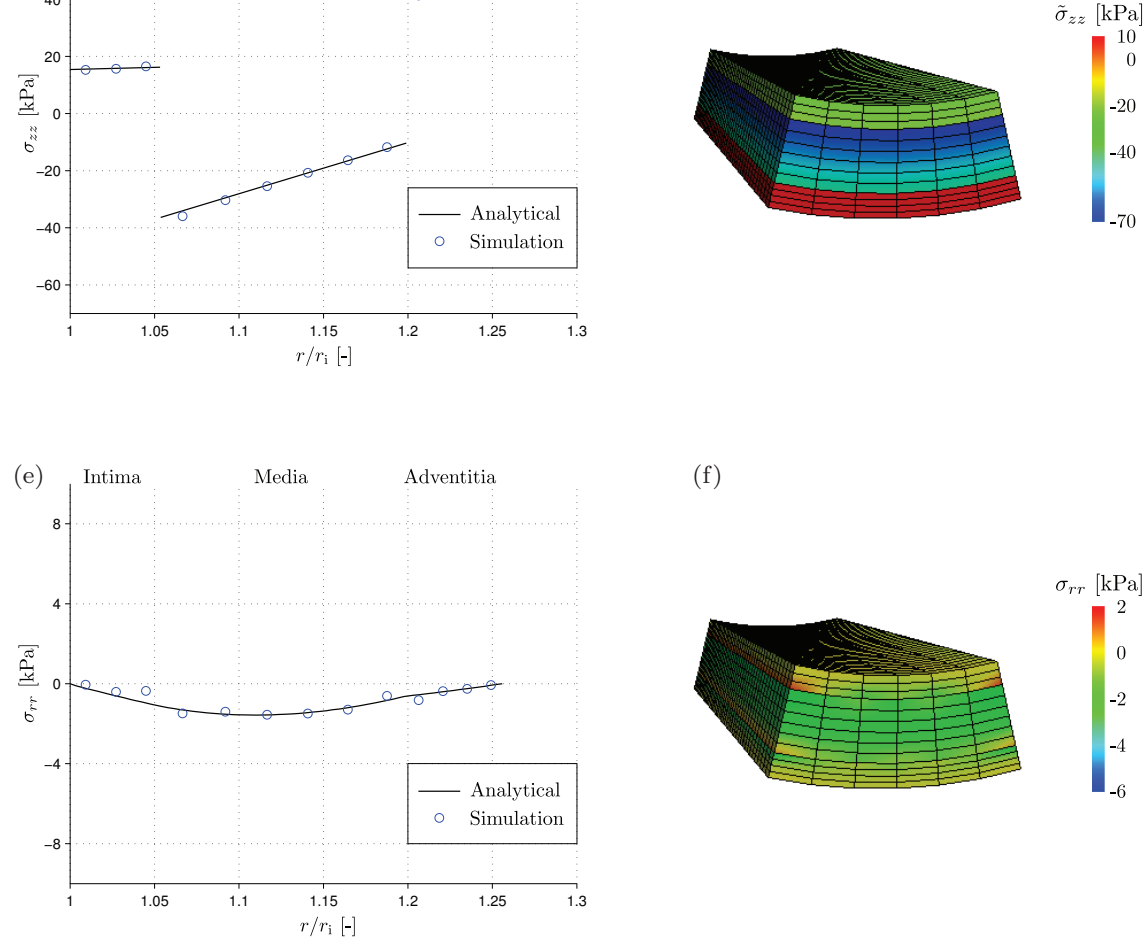

(f)

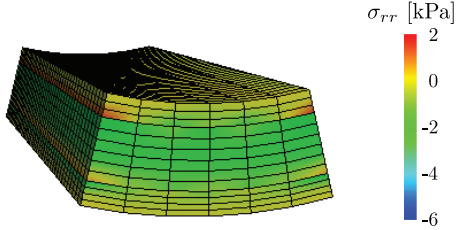

Figure 3: Three-dimensional stress components versus normalized radius for a three-layer arterial tissue sample in the unloaded, residually-stressed state, (a),(c),(e), and corresponding three-dimensional finite element representations, (b),(d),(f): circumferential stress $\sigma_{\theta \theta}$ (a),(b); equilibrium $\sigma_{z z}$ and simulated axial stresses $\tilde{\sigma}_{z z}(\mathrm{c}),(\mathrm{d})$; radial stress $\sigma_{r r}$ (e),(f). Following Holzapfel and Ogden [22], the axial stress in (c) is the equilibrium stress distribution $\sigma_{z z}=\tilde{\sigma}_{z z}-\bar{\sigma}_{z z}$, ensuring that the axial loads on the ends of the artery are zero, where $\tilde{\sigma}_{z z}$ are the FE simulated stresses and $\bar{\sigma}_{z z}$ is the mean axial stress. 


\section{3-D FE model.}

\subsection{Patient-Specific Analyses}

To demonstrate the efficacy of our methodology, we detail results from three representative patient-specific geometries (Patients 1,2,3; P1,P2,P3) for the three different types of simulations: (i) 'conventional'; (ii) 'prestressing'; (iii) 'novel'. To address the quality of the meshes, we detail the scaled Jacobian (which takes the range $[-1,1]$ ) results for the mesh of P1. Herein, all elements in the AAA wall have a scaled Jacobian value above 0.2 and $96.9 \%$ of the hexahedral elements have a value greater than 0.5 . In the ILT, all elements have scaled Jacobian values above 0.2 and $99.2 \%$ of the hexahedral elements have a value greater than 0.5 .

As a consequence of the types of simulations we obtain different individual displacement fields. The magnitude of the nodal displacement $\|\mathbf{u}\|$ provides information for comparison of the three types of simulations. At DBP $(85 \mathrm{mmHg})$, the maximum nodal displacement magnitude obtained using the conventional calculation is significant, while since we program both the prestressing and the novel calculations to maintain the imaged in vivo configuration under pressure loading to DBP, we observe no nodal displacements in these two types of simulations (not shown).

Subsequently, we increase the pressure load to SBP $(135 \mathrm{mmHg})$ and calculate substantially different distribution of $\|\mathbf{u}\|$ for the three different calculations. Figure 4 compares the distribution of the nodal displacement magnitude $\|\mathbf{u}\|$ obtained at SBP for P1 [(a),(b),(c); top row], P2 [(d),(e),(f); middle row] and P3 [(g),(h), (i); bottom row] using the three different types of simulations: conventional (first column), prestressing (middle column), novel (last column), the latter including both residual stresses and prestresses.

As another point for comparison, we focus on P1 and review the maximum nodal displacement magnitudes obtained at DBP and SBP using the three types of simulations. At DBP, $\|\mathbf{u}\|=$ $3.19 \mathrm{~mm}$ in the conventional calculation while, in both the prestressing and novel simulation types, we observe no nodal displacements $(\|\mathbf{u}\|=0 \mathrm{~mm})$ as expected. At SBP, $\|\mathbf{u}\|=3.53 \mathrm{~mm}$ in the conventional calculation, see Fig. 4(a), $1.29 \mathrm{~mm}$ in the prestressing calculation, see Fig. 4(b), 
(a)

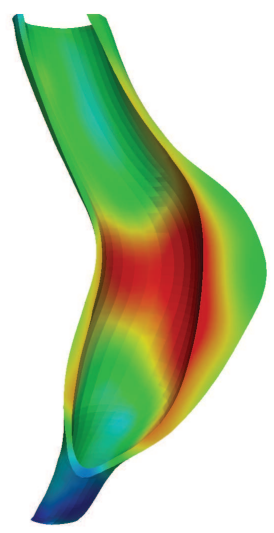

(d)

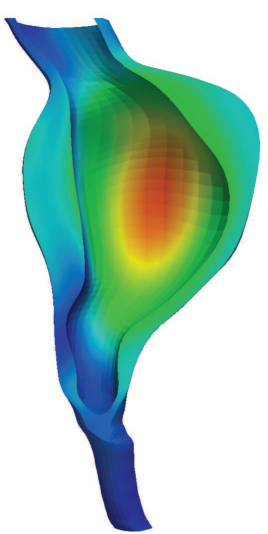

(g)

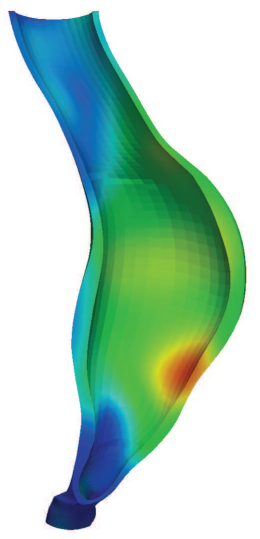

(b)

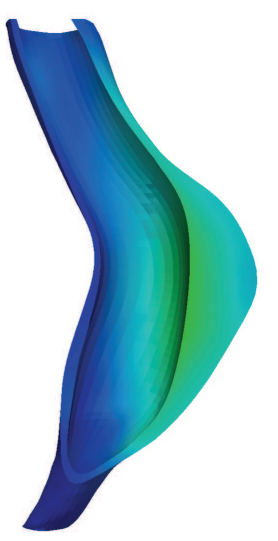

(e)

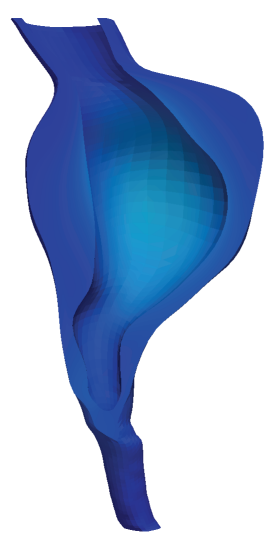

(h)

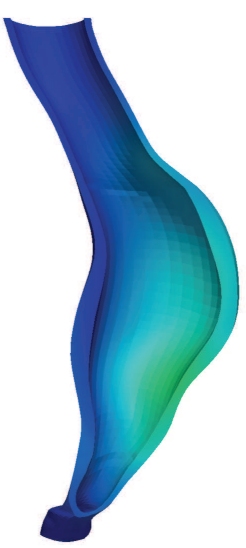

(c)

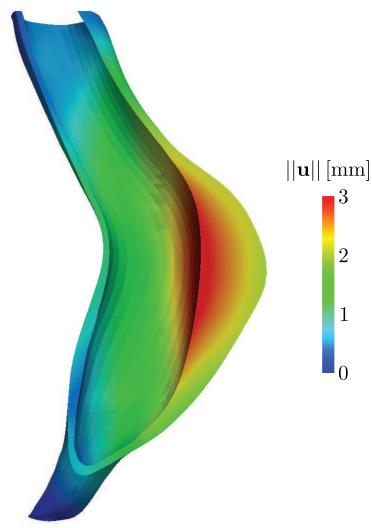

(f)

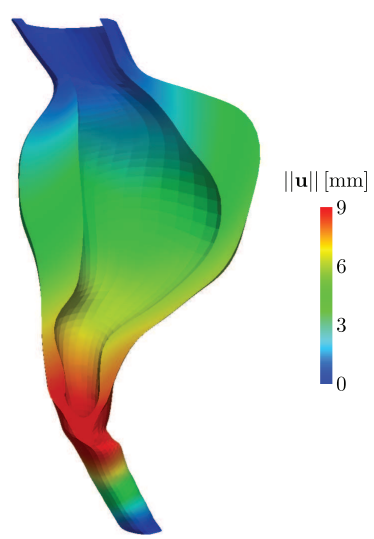

(i)

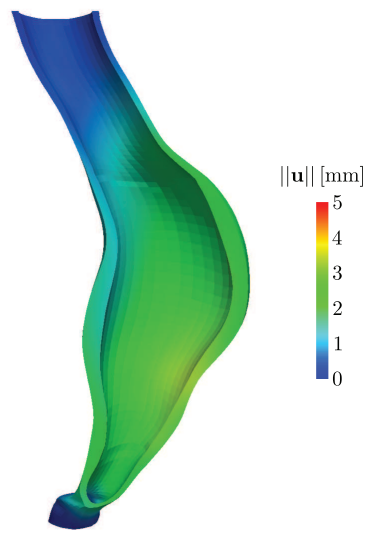

Figure 4: Representative finite element simulations showing the distribution of the nodal displacement magnitude $\|\mathbf{u}\|$ obtained at systolic blood pressure for Patient 1, (a),(b),(c), Patient 2, (d),(e),(f), and Patient 3, (g),(h),(i), using three different types of simulations: conventional, (a),(d),(g), prestressing (b),(e),(h), and novel, (c),(f),(i), the later accounting for both residual stresses and prestresses. 
and $3.10 \mathrm{~mm}$ in the novel calculation, see Fig. 4(c).

Following the same layout, Fig. 5 compares the distributions of circumferential Cauchy stresses $\sigma_{\theta \theta}$ obtained at DBP for P1 (a),(b),(c), P2 (d),(e),(f) and P3 (g),(h),(i) using the three different types of simulations: conventional, (a),(d),(g); prestressing, (b),(e),(h); novel, (c),(f),(i). Similarly, Fig. 6 compares the distributions of circumferential Cauchy stresses $\sigma_{\theta \theta}$ obtained at SBP. For closer inspection at SBP, Fig. 7 7 compares presumably healthy aortic cross-sections arbitrarily cut from the FE models above the thrombi but sufficiently far from the top boundary planes in Fig. 6 Therein, Fig. 7 compares the distribution of the circumferential Cauchy stress $\sigma_{\theta \theta}$ obtained at SBP for P1 (a),(b),(c), P2 (d),(e),(f) and P3 (g),(h),(i) using the three different types of simulations: conventional, (a),(d),(g); prestressing, (b),(e),(h); novel, (c),(f),(i).

\section{Discussion}

Holzapfel et al. [28] demonstrated that a single parameter such as the opening angle is not sufficient to characterize the complex, multi-layered 3-D residual stress field present in arterial tissues, hence the motivation for our current work. Separating the human intima, media and adventitia experimentally, i.e., a multi-layered stress release, allows the tissue segments to get much closer to true stress-free states where the 3-D residual stretches and curvatures can be measured.

To our knowledge, the methodology presented here is the first to include 3-D residual stress distributions with such fidelity into FE models, where we account for the effects of both residual stretches and residual curvatures determined experimentally using human tissues. We include the arterial wall thickness estimated from medical images in our patient-specific simulations, we model the three layers of the aorta explicitly based on experimentally determined through-the-

thickness ratios of intima/media/adventitia, we account for both layer-specific residual stresses and prestresses in the imaged configuration in order to maintain theoretical consistency, and we use both layer-specific material parameters and residual stretches determined experimentally from human tissues. 
(a)

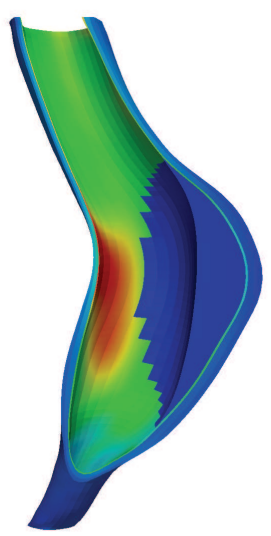

(d)

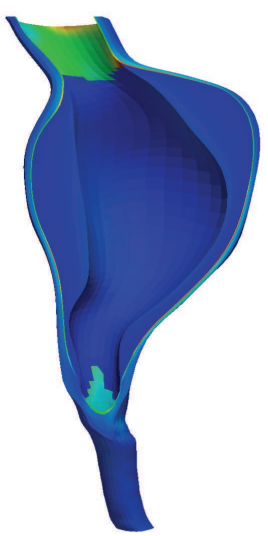

(g)

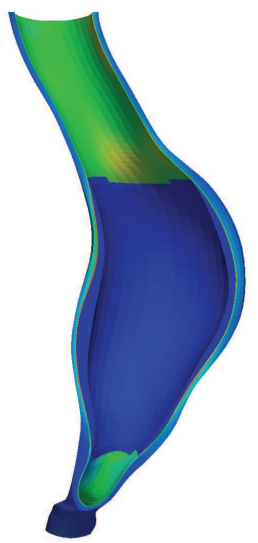

(b)

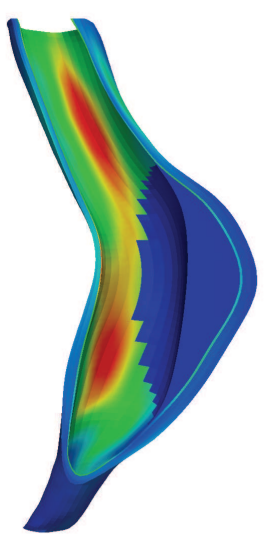

(e)

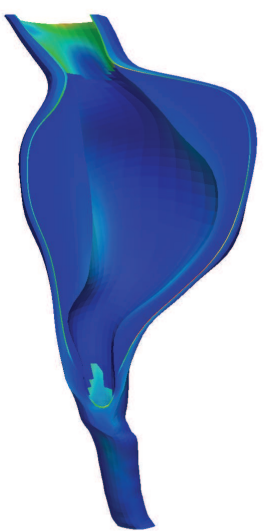

(h)

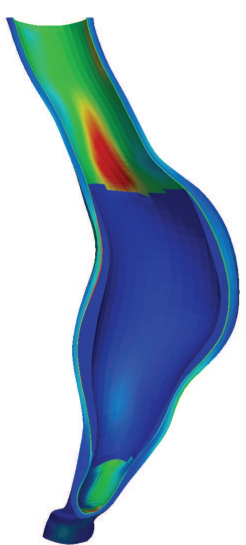

(c)

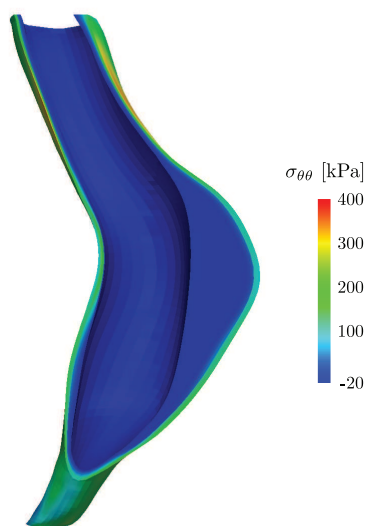

(f)

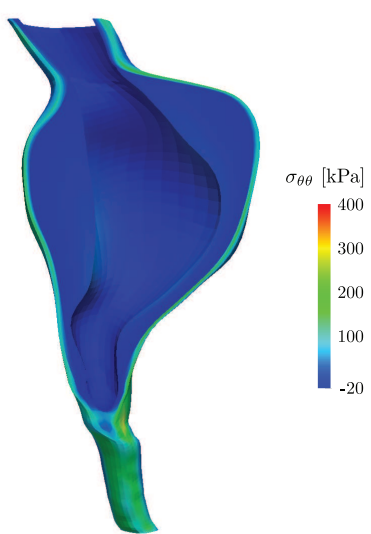

(i)

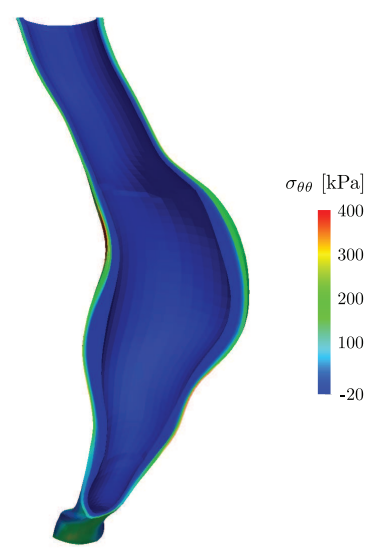

Figure 5: Representative finite element simulations showing the distribution of the circumferential Cauchy stress $\sigma_{\theta \theta}$ obtained at diastolic blood pressure for Patient 1, (a),(b),(c), Patient 2, (d),(e),(f), and Patient 3, $(\mathrm{g}),(\mathrm{h}),(\mathrm{i})$, using three different types of simulations: conventional, (a),(d),(g), prestressing (b),(e),(h), and novel, (c),(f),(i), the later accounting for both residual stresses and prestresses. 
(a)

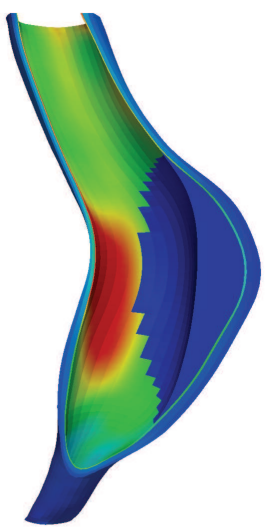

(d)

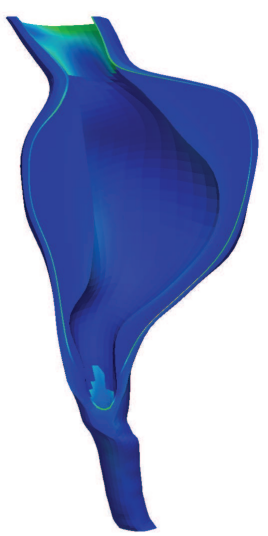

(g)

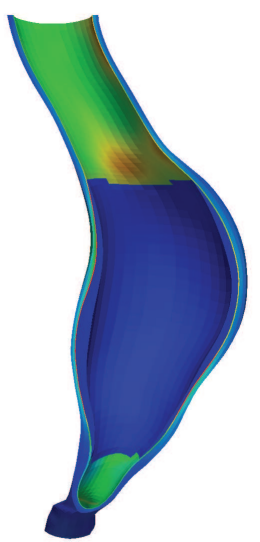

(b)

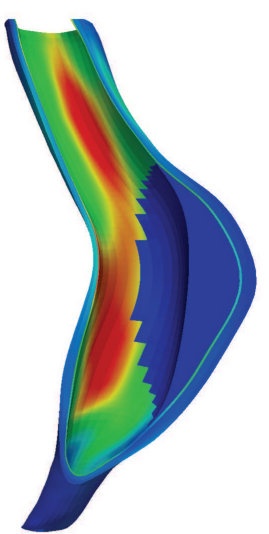

(e)

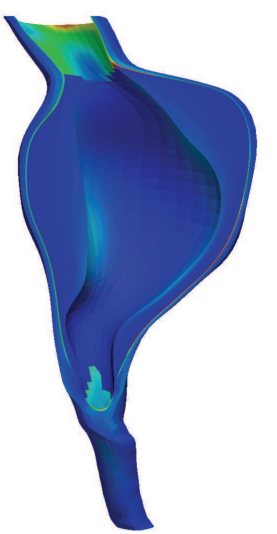

(h)

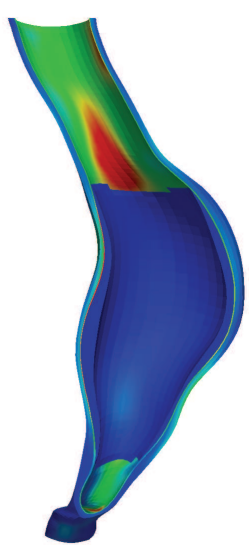

(c)

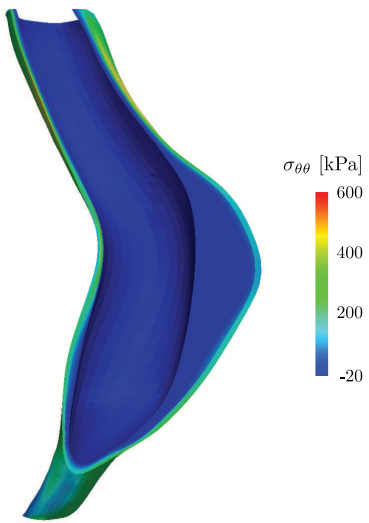

(f)

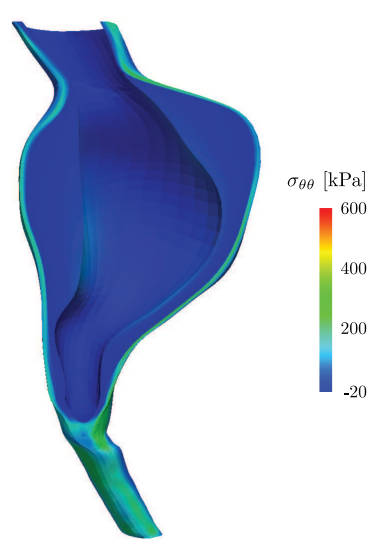

(i)

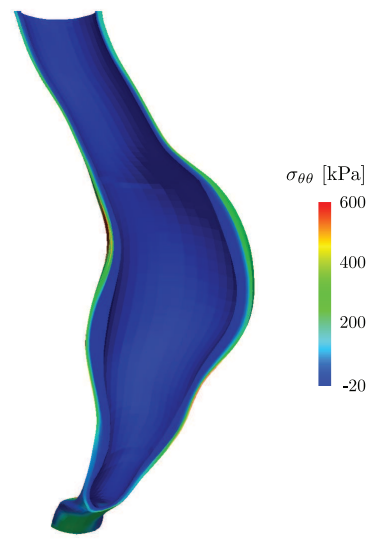

Figure 6: Representative finite element simulations showing the distribution of the circumferential Cauchy stress $\sigma_{\theta \theta}$ obtained at systolic blood pressure for Patient 1, (a),(b),(c), Patient 2, (d),(e),(f), and Patient 3, (g),(h),(i), using three different types of simulations: conventional, (a),(d),(g), prestressing (b),(e),(h), and novel, (c),(f),(i), the later accounting for both residual stresses and prestresses. 


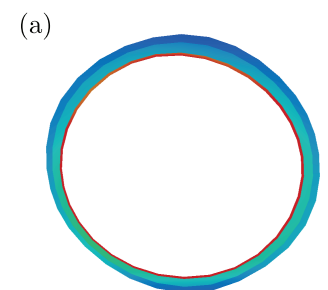

(d)

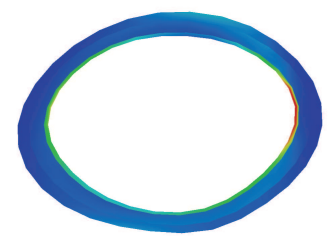

(g)

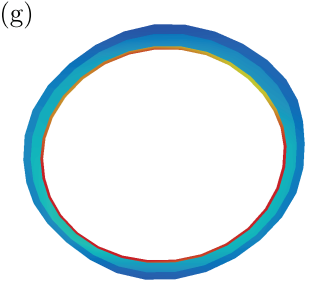

(b)

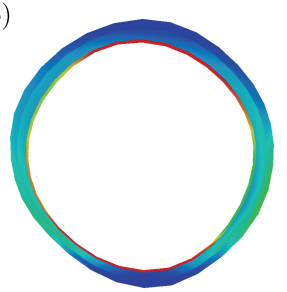

(e)

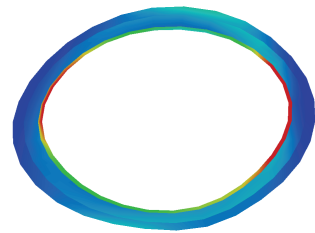

(h)

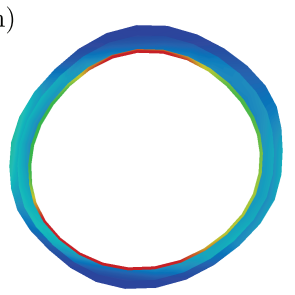

(c)

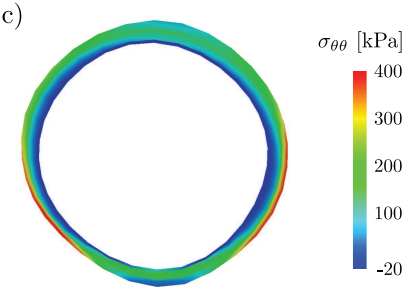

(f)

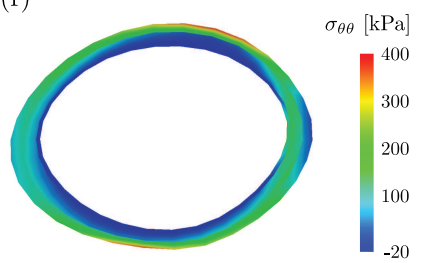

(i)

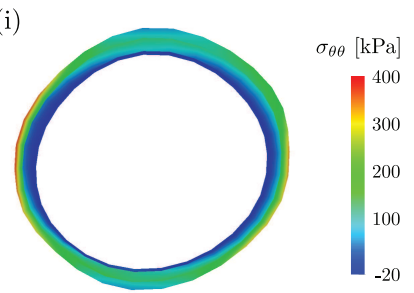

Figure 7: Representative finite element results for presumably healthy aortic rings showing the distribution of the circumferential Cauchy stress $\sigma_{\theta \theta}$ obtained at systolic blood pressure for Patient 1, (a),(b),(c), Patient 2, (d),(e),(f), and Patient 3, (g),(h),(i), using three different types of simulations: conventional, (a),(d),(g), prestressing, (b),(e),(h), and novel, (c),(f),(i), the last accounting for both residual stresses and prestresses.

\subsection{Validation Analyses}

The validation test case shows an excellent match between the analytical results from Holzapfel and Ogden [22] and our simulation results (cf., Fig. 3) and demonstrates both the correct implementation of our method, as well as its accuracy. This test case provides an additional check on our modeling assumptions. In our numerical reconstruction of the axial tissue strip we include both the circumferential and axial curvatures measured experimentally. However, Holzapfel and Ogden [22] consider the larger of these two curvatures in order to derive a closed-form, analytical expression for the deformation gradients $\mathbf{F}_{\mathrm{RS}}^{(i)}$, with $i \in[\mathrm{I}, \mathrm{M}, \mathrm{A}]$. Since our analytical expressions 
$\mathbf{F}_{\mathrm{RS}}^{(i)}\left(r^{(i)}\right)$ in (1)-(5) are based on [22], we also consider the largest curvature. The results in Fig. 3 demonstrate that neglecting the bending stresses resulting from the smaller residual curvature does not cause a significant error. Residual stresses generated from the smaller residual curvature likely explain the small differences between the analytical result and our simulation result for the circumferential stress distribution in the media (cf., Fig. 3 a)).

\subsection{Patient-Specific Analyses}

Discussing details of our patient-specific analyses, we employ an MRI protocol that we have specifically tuned to enhance contrast between the aortic wall and the surrounding tissue. The improved protocol allows us to segment both the aortic lumen and the outer edge of the aorta, and thus locally estimate the true thickness of the aortic wall. The importance of estimating the arterial wall thickness, and hence including non-uniform wall thicknesses to FE models, is discussed in Humphrey and Holzapfel [46]. In general, MRI allows for improved tissue contrast versus computed tomography angiography, which enables assessment of thrombus consistency as well [47].

Our custom meshing algorithm, detailed in Tarjuelo-Gutiérrez et al. [35], allows us to control the number and distribution of hexahedral elements. With this algorithm we have tuned the FE simulations such that we achieve both solution convergence and a suitable distribution of suitable elements based on the scaled Jacobian as a quality measure. Additionally, we calculate relatively smooth stress distributions even when plotting element stresses (not shown). Hence, we believe the meshes are sufficient to accurately resolve the stress distributions we present.

Comparing the three types of solutions across Patients 1,2 and 3 we notice the influence of residual stretches/stresses in all solutions. The overall deformation field changes significantly for the three different types of simulations, as depicted in Fig. 4 We predict large nodal displacement magnitudes for the conventional calculation at DBP and only a relatively small increase at SBP. This behavior is explained by the exponential stiffening of the collagen fibers in the AAA wall, enabling large displacements only at relatively low loads. For the prestressing and novel calculations at DBP, we observe no displacements, indicating correct implementation of the GPA 
(i.e., we maintain the imaged in vivo geometry). However, we obtain a large difference in nodal displacement magnitudes for both algorithms at SBP. Circumferential stresses estimated in the intima at diastole with the prestressing calculation are relatively high, suggesting the presence of pronounced stiffening. Conversely, in the novel calculation these stresses are relatively moderate in the tissue layers. Thus, the additional pressure increase from DBP to SBP has a larger impact on nodal displacement magnitudes in the novel calculation. When comparing nodal displacements across the three simulation types, these are largest in the conventional calculation, consistent with findings in the literature, e.g., [27].

Reviewing the results more closely, there is a clear difference in the stress distributions, especially in the maximum stresses, between the conventional calculations and the calculations including initial stresses (prestresses or residual stresses and prestresses), as shown at $85 \mathrm{mmHg}$ in the Figs. 5(a),(d),(g) versus (b),(e),(h) or (c),(f),(i) and at $135 \mathrm{mmHg}$ in the Figs. 6(a),(d),(g) versus (b),(e),(h) or (c),(f),(i). Our results indicate that accounting for initial stresses within patientspecific simulations increases both the distribution range and maximum of the circumferential Cauchy stresses. We postulate that this effect is due to larger local curvatures (inverse of the local radius) in the imaged geometry versus the deformed geometry of the conventional calculation [20].

Continuing on to the stress results, a quantitative comparison of the circumferential stress distribution $\sigma_{\theta \theta}$ obtained applying the three types of simulations at DBP, as depicted in Fig. 5, suggests that the predicted mean stress magnitude in the intima using the conventional and prestressing calculations are non-physiological and unrealistically high. This is in contrast to observed mean stresses in the media and adventitia, where stress estimates seem to be very low and essentially non-existent. The novel algorithm, including both residual stresses and prestresses, applied to patient-specific FE simulations of arterial tissue provides reasonable mean stress magnitudes in the circumferential direction for the media and the adventitia, cf., Humphrey and Holzapfel [46]. However, stress estimates in the intima may be too low as we observe compressive mean circumferential stresses.

Upon loading the AAAs to SBP we observe a dramatic increase in the mean circumferential 
stress values in the intima when applying both the conventional and prestressing calculations, again due to exponential stiffening of the collagen fibers in the high load regime, as depicted in Fig. 6. However, we observe only minor stress increases in the media and adventitia as a consequence. Here the intima appears to 'stress-shield' the media and adventitia, leading to their moderate increases in stresses at SBP. Conversely, the novel calculation predicts more reasonable mean stress values in the media and adventitia. We also observe increased, but still (slightly) negative, circumferential stresses in the intima, see Figs.6(c),(f),(i). Compressive circumferential stresses (hoop stresses) at the inner boundary of the arterial wall are reported in the literature when prestressing algorithms and residual stresses are combined, e.g., Alastrué et al. [27,48].

For closer inspection at SBP, Fig. 7 / compares presumably healthy aortic cross-sections arbitrarily cut from the FE models above the thrombi but sufficiently far from the top boundary planes in Fig. 6. Here the circumferential Cauchy stress distribution $\sigma_{\theta \theta}$ seems to be overall more uniform through the tissue thickness (i.e., smaller stress gradients in the local radial directions) in the novel calculation, Figs. 7(c),(f),(i), compared to both the conventional, Figs. 7(a),(d),(g), and the prestressing calculations, Figs. 7b),(e),(h). Nonetheless, in all three types of simulations we observe large variations in the circumferential stress values $\sigma_{\theta \theta}$, on the order of $100 \mathrm{kPa}$, at both pressures (DBP, SBP). Such large variations have also been shown in other studies with patientspecific geometries of arteries, see, e.g., [8, 12, 26, 27, 49, 52], but such circumferential variations of $\sigma_{\theta \theta}$ in these results is never discussed in detail.

Such results are a consequence of both the patient-specific geometry, where the tissue geometry and thickness is estimated from MRI, and the use of homogeneous material parameters within each tissue layer, however this is not plausible under physiological loads. Experiments report that the stiffness of aortic tissue varies along the circumferential direction [53, 54], and suggest that remodeling in healthy arteries aims at maintaining a nearly constant wall stress, e.g., by changing the wall thickness or the composition of constituents [55]. Further experiments confirm that wall stiffness and strength correlate with wall thickness in AAAs [56]. Finally, the high stress variation contradicts growth and remodeling theories that assume deviation from a uniform homeostatic stress is the principal driver of tissue adaptation [17, 57]. 
Remarkable differences in the lumen shape are observed when we compare the conventional calculation to the prestressing and novel calculations (Fig. 7). We obtain relatively smooth curvatures in the conventional calculation, in contrast to the prestressing and novel (geometry preserving approaches) calculations, where large curvature differences are still present and possibly explaining the large stress variations. Experimental investigations aimed at determining local variations in both material parameters [54] and residual stresses [58] would help clarify our results, as these will likely homogenize the overall state of stress.

The novel calculation, in which prestresses and residual stresses are included, leads to physiologically relevant mean stress values for the media and adventitia at DBP and SBP, however, we observe a small compressive stress in the circumferential direction of the intima. The reason for this non-physiological stress estimate might be an over-prediction of residual stretches in the analytical calculation performed prior to FE simulation. To rectify this short-coming, it is possible to adjust the deformation gradient mapping the 'nearly stress-free' reference configuration for the separated medial layer to the unloaded, patient-specific geometry $\left(\mathbf{F}_{\mathrm{RS}}^{(\mathrm{M})}\left(r^{(\mathrm{M})}\right)\right.$; (2) $)$ using a constant 'adjustment' factor. We explored this possibility with a parameter study aimed to determine the influence of residual stretches on the mean circumferential stress $\sigma_{\theta \theta}$, and it is possible to achieve the expected positive mean stress values in the intima at DBP. However, traction continuity at the intima-media and the media-adventitia interface may be lost when applying such an adjustment factor. Additionally, this is a purely phenomenological approach, unfounded in experimental results, and thus is here not pursued further.

Results obtained for all types of simulations indicate a state of hydrostatic compression in the ILT. However, the material model and experimentally fitted parameters applied here (cf., [32]) were determined under biaxial extension and the mechanical behavior of the individual ILT layers under compression remains unknown. Thus, the constitutive model used may not be an adequate representation of the tissue composite in compression and stress results should be treated with caution.

Raghavan et al. [12] studied the effect of residual stresses using the opening angle method and FE analysis. Therein, the authors compared the circumferential and radial stress distributions in 
patient-specific arterial segments to those estimated on cylindrical approximations of the same arterial segments. They conclude that the residual stress distributions do not vary significantly in these two cases. The results in [12] provide confirmation that mapping the analytically determined residual stretches into patient-specific geometries will still lead to a 'best estimate' of the true patient-specific residual stretches.

In general, our novel calculation has the potential to increase the reliability of stress predictions within AAA walls, and the overall mean stresses of the individual arterial layers become substantially more homogeneous, as expected, at higher loads such as SBP. The 3-D residual stresses influence the resulting stress distribution, reducing the stresses in the intima by shifting load to the media and adventitia. The result is a more homogenous stress distribution through the thickness of the aorta. Many researchers have concluded that residual stresses have a significant effect on the in vivo distribution of stresses and should tend to homogenize these distributions, cf., e.g., [4,21].

Finally, our methodology for mapping the residual deformation gradients $\mathbf{F}_{\mathrm{RS}}^{(i)}\left(r^{(i)}\right)$, with $i \in$ $[\mathrm{I}, \mathrm{M}, \mathrm{A}]$, (1)-(5), applies to virtually any FE mesh, and is not restricted to the well-structured meshes used in our analysis.

\subsection{Limitations}

Advanced mechanical modeling of complex 3-D biomechanical structures such as AAAs presents several challenges, and includes several assumptions and limitations influencing the results. In the analysis performed to validate our novel calculation for including residual stresses into patient-specific FE simulations we assume that the reference configurations of the separated layers, which are nearly stress-free, are completely stress-free. It is likely that additional cuts through the arterial tissue may be necessary to obtain zero-stress states [58]. In fact, the arterial wall would theoretically need to be separated into infinitesimally small parts to obtain configurations truly free from residual stresses [59,60].

Furthermore, the reference 'stress-free' geometry is a simplification of the real, experimentally- 
determined configurations using samples from 11 patients [28]. Determination of curvatures in the axial and circumferential directions was performed by approximating the actual geometry by circular arcs [28]. Holzapfel et al. [28] approximated complex curvatures in both directions with circular arcs, and assumed stretches to be uniform within each layer. The circularity assumption was investigated in the literature and confirmed to be reasonable [12].

The analytically determined residual stretches calculated in the preprocessing stage of the proposed method (cf., [22]) are also based on modeling the aortic tissue as a neo-Hookean material to make the analysis tractable. Here the residual stretches are likely small and thus the influence of stiffening of collagen fibers is neglected. Thus, the calculated residual deformations in (1)-(5) could be overestimated.

Performing the residual stress experiments includes the excision, i.e. unloading, of the artery from the human cadaver and subsequently cutting of axial and circumferential tissue strips [28]. As a consequence, residual stresses are released, leading to an equilibrium configuration after some time for relaxation. This experiment indicates that residual stretches are measured relative to the unloaded geometry, and thus should be included in the unloaded artery. In our calculation we include residual stresses into the loaded geometry of the artery, that determined from MR Imaging at DBP, prior to application of the pressure loading via the GPA. The impact of this geometry mismatch on the predicted stress states was not quantified.

Our patient-specific FE analyses treat the AAA wall as a healthy abdominal aorta, assuming that the three-layered structure and collagen fiber alignments of the tissue are not compromised. However, diseased AAA walls, particularly those covered by ILTs, are degenerated and fundamentally different to healthy human abdominal aortas in terms of morphology, collagen fiber orientations and material properties [32,61]. Furthermore, lipid pools and calcifications may be present in AAAs. We do not segment these structural components during image processing and therefore do not consider them in our FE simulations, although these details can be added, cf., [44, 62].

Experimental data on the residual stresses in diseased arterial tissues do not exist, and thus it remains unknown to what extent the formation and presence of ILT changes these residual stresses. Lacking data we assume here that this influence is negligible and we use the available residual 
stress data from healthy abdominal aortas. Here, in light of the degenerated AAA tissues, the residual stresses could be overestimated. Experimental data on the residual stresses present in the ILT is also lacking. Based on unpublished observations made at the Institute of Biomechanics, Graz University of Technology, residual stresses in the individual ILT layers are relatively small and are therefore not included in our FE analyses. Here the residual stresses could be underestimated.

\subsection{Concluding Remarks}

Our proposed methodology simultaneously accounts for experimentally determined residual stresses and in vivo loading conditions on patient-specific simulations of aortas. Including residual stresses in patient-specific FE simulations of aortic tissues significantly impacts both the stress distributions within the tissue (and its layers) and the global (organ-level) deformation, and is thus important to consider.

We apply our novel approach for including residual stresses into patient-specific FE models of aortic tissues to examples on AAAs. Our methodology is equally useful for incorporating residual stresses into the ILT although residual stresses within the ILT have not yet been studied experimentally, and hence no data are available for calibration.

Overall our methodology applies to any residually stressed material where experimental data on the residual deformations are available. Such methods, coupled with appropriate experimental data, aim at increasing the accuracy of FE analyses for patient-specific studies in computational biomechanics and may lead to increased clinical application of simulation tools. In the future such simulations of arteries of individual patients may aid clinicians to diagnose and treat arterial pathologies. 


\section{Acknowledgment}

We gratefully acknowledge the financial support of the European Commission under the 7th Framework Programme in the scope of the project SCATh - Smart Catheterization, Grant Agreement Number 248782 and Hannah Weisbecker, Institute of Biomechanics, TU Graz, for help with the Generalized Prestressing Algorithm.

\section{References}

[1] Y. C. Fung. On the foundations of biomechanics. J. Appl. Mech., 50:1003-1009, 1983.

[2] R. N. Vaishnav and J. Vossoughi. Estimation of residual strains in aortic segments. In C. W. Hall, editor, Biomedical Engineering II: Recent Developments, pages 330-333. Pergamon Press, New York, 1983.

[3] C. J. Chuong and Y. C. Fung. Three-dimensional stress distribution in arteries. J. Biomech. Eng., 105:268-274, 1983.

[4] C. J. Chuong and Y. C. Fung. On residual stress in arteries. J. Biomech. Eng., 108:189-192, 1986.

[5] Y. C. Fung. What are the residual stresses doing in our blood vessels? Ann. Biomed. Eng., 19:237-249, 1991.

[6] R. N. Vaishnav and J. Vossoughi. Residual stress and strain in aortic segments. J. Biomech., 20:235-239, 1987.

[7] T. Matsumoto and K. Hayashi. Stress and strain distribution in hypertensive and normotensive rat aorta considering residual strain. J. Biomech., 118:62-73, 1996.

[8] A. Delfino, N. Stergiopulos, J. E. Moore Jr., and J.-J. Meister. Residual strain effects on the stress field in a thick wall finite element model of the human carotid bifurcation. $J$. Biomech., 30:777-786, 1997. 
[9] G. A. Holzapfel, T. C. Gasser, and R. W. Ogden. A new constitutive framework for arterial wall mechanics and a comparative study of material models. J. Elasticity, 61:1-48, 2000.

[10] S. J. Peterson and R. J. Okamoto. Effect of residual stress and heterogeneity on circumferential stress in the arterial wall. J. Biomech. Eng., 122:454-456, 2000.

[11] J. D. Humphrey. Cardiovascular Solid Mechanics. Cells, Tissues, and Organs. SpringerVerlag, New York, 2002.

[12] M. L. Raghavan, S. Trivedi, A. Nagaraj, D. D. McPherson, and K. B. Chandran. Threedimensional finite element analysis of residual stress in arteries. Ann. Biomed. Eng., 32:257-263, 2004.

[13] K. Takamizawa and K. Hayashi. Strain energy density function and uniform strain hypothesis for arterial mechanics. J. Biomech., 20:7-17, 1987.

[14] S. Q. Liu and Y. C. Fung. Relationship between hypertension, hypertrophy, and opening angle of zero-stress state of arteries following aortic constriction. J. Biomech. Eng., 111:325-335, 1989.

[15] E. K. Rodriguez, A. Hoger, and A. D. McCulloch. Stress-dependent finite growth in soft elastic tissues. J. Biomech., 27:455-467, 1994.

[16] L. A. Taber. Biomechanics of growth, remodelling, and morphognesis. Appl. Mech. Rev., 48:487-543, 1995.

[17] L. A. Taber and D. W. Eggers. Theoretical study of stress-modulated growth in the aorta. J. Theor. Biol., 180:343-357, 1996.

[18] A. Rachev. Theoretical study of the effect of stress-dependent remodeling on arterial geometry under hypertensive conditions. J. Biomech., 30:819-827, 1997.

[19] M. W. Gee, Ch. Förster, and W. A. Wall. A computational strategy for prestressing patientspecific biomechanical problems under finite deformation. Int. J. Numer. Meth. Biomed. Engng., 26:52-72, 2009. 
[20] H. Weisbecker, D. M. Pierce, and G. A. Holzapfel. A generalized prestressing algorithm for finite element simulations of pre-loaded geometries with application to the aorta. Int. J. Numer. Method Biomed. Eng., 30:857-872, 2014.

[21] R. Bustamante and G. A. Holzapfel. Methods to compute 3d residual stress distributions in hyperelastic tubes with application to arterial walls. Int. J. Eng. Sci., 48:1066-1082, 2010.

[22] G. A. Holzapfel and R. W. Ogden. Modelling the layer-specific 3D residual stresses in arteries, with an application to the human aorta. J. R. Soc. Interface, 7:787-799, 2010.

[23] J.-S. Ren. Growth and residual stresses of arterial walls. J. Theor. Biol., 337:80-88, 2013.

[24] J. Schröder and S. Brinkhues. A novel scheme for the approximation of residual stresses in arterial walls. Archive Appl. Mech., 84:881-898, 2014.

[25] T. Waffenschmidt and A. Menzel. Extremal states of energy of a double-layered thickwalled tube - application to residually stressed arteries. J. Mech. Beh. Biomed. Mat., 29:635-654, 2014.

[26] V. Alastrué, E. Peña, M. Á. Martínez, and M. Doblaré. Assessing the use of the "Opening Angle Method" to enforce residual stresses in patient-specific arteries. Ann. Biomed. Eng., 35:1821-1837, 2007.

[27] V. Alastrué, A. Garía, E. Peña, J. F. Rodríguez, M. A. Martínez, and M. Doblaré. Numerical framework for patient-specific computational modelling of vascular tissue. Int. J. Numer. Meth. Engng, 26:35-51, 2010.

[28] G. A. Holzapfel, G. Sommer, M. Auer, P. Regitnig, and R. W. Ogden. Layer-specific 3D residual deformations of human aortas with non-atherosclerotic intimal thickening. Ann. Biomed. Eng., 35:530-545, 2007.

[29] T. C. Gasser and G. A. Holzapfel. 3D crack propagation in unreinforced concrete. A twostep algorithm for tracking 3D crack paths. Comput. Meth. Appl. Mech. Eng., 195:5198$5219,2006$. 
[30] G. A. Holzapfel, G. Sommer, C. T. Gasser, and P. Regitnig. Determination of the layerspecific mechanical properties of human coronary arteries with non-atherosclerotic intimal thickening, and related constitutive modelling. Am. J. Physiol. Heart Circ. Physiol., 289:H2048-2058, 2005.

[31] H. Weisbecker, D. M. Pierce, P. Regitnig, and G. A. Holzapfel. Layer-specific damage experiments and modeling of human thoracic and abdominal aortas with non-atherosclerotic intimal thickening. J. Mech. Beh. Biomed. Mat., 12:93-106, 2012.

[32] J. Tong, T. Cohnert, P. Regitnig, and G. A. Holzapfel. Effects of age on the elastic properties of the intraluminal thrombus and the thrombus-covered wall in abdominal aortic aneurysms: biaxial extension behavior and material modeling. Eur. J. Vasc. Endovasc. Surg., 42:207219, 2011.

[33] FEAP - A Finite Element Analysis Program, Version 8.2 User Manual. University of California at Berkeley, Berkeley, California, 2008.

[34] G. A. Holzapfel. Determination of material models for arterial walls from uniaxial extension tests and histological structure. J. Theor. Biol., 238:290-302, 2006.

[35] J. Tarjuelo-Gutierrez, B. Rodriguez-Vila, D. M. Pierce, T. E. Fastl, P. Verbrugghe, I. Fourneau, G. Maleux, P. Herijgers, G. A. Holzapfel, and E. J. Gómez. High-quality conforming hexahedral meshes of patient-specific abdominal aortic aneurysms including their intraluminal thrombi. Med. Biol. Eng. Comput., 52:159-168, 2014.

[36] O. C. Zienkiewicz, R. L. Taylor, and J. Z. Zhu. The Finite Element Method: Its Basis and Fundamentals. Butterworth-Heinemann, Oxford, 2005.

[37] T. C. Gasser, R. W. Ogden, and G. A. Holzapfel. Hyperelastic modelling of arterial layers with distributed collagen fibre orientations. J. R. Soc. Interface, 3:15-35, 2006.

[38] A. J. Schriefl, G. Zeindlinger, D. M. Pierce, P. Regitnig, and G. A. Holzapfel. Determination of the layer-specific distributed collagen fiber orientations in human thoracic and abdominal aortas and common iliac arteries. J. R. Soc. Interface, 9:1275-1286, 2012. 
[39] G. De Santis, M. De Beule, K. Van Canneyt, P. Segers, P. Verdonck, and B. Verhegghe. Fullhexahedral structured meshing for image-based computational vascular modeling. Med. Eng. Phys., 33:1318-1325, 2011.

[40] A Henderson Squillacote. The ParaView Guide. A Parallel Visualization Application. Kitware Inc., 2007.

[41] C. J. Stimpson, C. D. Ernst, P. Knupp, P. P. Pébay, and D. Thompson. The Verdict Geometric Quality Library. Sandia National Laboratories, 2007.

[42] G. A. Holzapfel. Nonlinear Solid Mechanics. A Continuum Approach for Engineering. John Wiley \& Sons, Chichester, 2000.

[43] G. A. Holzapfel, M. Stadler, and T. C. Gasser. Changes in the mechanical environment of stenotic arteries during interaction with stents: Computational assessment of parametric stent design. J. Biomech. Eng., 127:166-180, 2005.

[44] D. E. Kiousis, S. F. Rubinigg, M. Auer, and G. A. Holzapfel. A methodology to analyze changes in lipid core and calcification onto fibrous cap vulnerability: The human atherosclerotic carotid bifurcation as an illustratory example. J. Biomech. Eng., 131:121002, 2009.

[45] A. V. Chobanian, G. L. Bakris, H. R. Black, W. C. Cushman, L. A. Green, J. L. Izzo, D. W. Jones, B. J. Materson, S. Oparil, J. T. Wright, and E. J. Roccella. The seventh report of the joint national committee on prevention, detection, evaluation and treatment of high blood pressure. J. Am. Med. Assoc., 289:2560-2572, 2003.

[46] J. D. Humphrey and G. A. Holzapfel. Mechanics, mechanobiology, and modeling of human abdominal aorta and aneurysms. J. Biomech., 45:805-814, 2012.

[47] S. Haulon, C. Lions, E. McFadden, M. Koussa, V. Gaxotte, P. Halna, and J. Beregi. Prospective evaluation of magnetic resonance imaging after endovascular treatment of infrarenal aortic aneurysms. Eur. J. Vasc. Endovasc. Surg., 22:62-69, 2001. 
[48] V. Alastrué, J. F. Rodríguez, B. Calvo, and M. Doblaré. Structural damage models for fibrous biological soft tissues. Int. J. Solids Structures, 44:5894-5911, 2007.

[49] D. F. Elger, R. S. Blackketter, R. S. Budwig, and K. H. Johansen. The influence of shape on the stresses in model abdominal aortic aneurysms. J. Biomed. Eng., 118:326-332, 1996.

[50] M. S. Sacks, D. A. Vorp, M. L. Raghavan, M. P. Federle, and M. W. Webster. In vivo three-dimensional surface geometry of abdominal aortic aneurysms. Ann. Biomed. Eng., 27:469-479, 1999.

[51] S. de Putter, B. J. Wolters, M. C. Rutten, M. Breeuwer, F. A. Gerritsen, and F. N. van de Vosse. Patient-specific initial wall stress in abdominal aortic aneurysms with a backward incremental method. J. Biomech., 40:1081-1090, 2007.

[52] L. Speelman, E. M. H. Bosboom, G. W. Schurink, J. Buth, M. Breeuwer, M. J. Jacobs, and F. N. van de Vosse. Initial stress and nonlinear material behavior in patient-specific AAA wall stress analysis. J. Biomech., 42:1713-1719, 2009.

[53] M. A. Nicosia, J. S. Kasalko, R. P. Cochran, D. R. Einstein, and K. S. Kunzelman. Biaxial mechanical properties of porcine ascending aortic wall tissue. J. Heart Valve Dis., 11:680686, 2002.

[54] J. Kim and S. Baek. Circumferential variations of mechanical behavior of the porcine thoracic aorta during the inflation test. J. Biomech., 44:1941-1947, 2011.

[55] T. Matsumoto and K. Hayashi. Mechanical and dimensional adaptation of rat aorta to hypertension. J. Biomech. Eng., 116:278-283, 1994.

[56] C. Reeps, A. Maier, J. Pelisek, F. Härtl, V. Grabher-Meier, W. Wall, M. Essler, H.-H. Eckstein, and M. Gee. Measuring and modeling patient-specific distributions of material properties in abdominal aortic aneurysm wall. Biomech. Model. Mechanobiol., 12:717-733, 2013. 
[57] J. D. Humphrey. Vascular adaptation and mechanical homeostasis at tissue, cellular, and sub-cellular levels. Cell Biochem. Biophys., 50:53-78, 2008.

[58] S. Q. Liu and Y. C. Fung. Zero-stress states of arteries. J. Biomech. Eng., 110:82-84, 1988.

[59] A. Rachev and S. E. Greenwald. Residual strains in conduit arteries. J. Biomech., 36:661670, 2003.

[60] A. Klarbring, T. Olsson, and J. Stålhand. Theory of residual stresses with application to an arterial geometry. Arch. Mech., 59:341-364, 2007.

[61] J. P. Vande Geest, M. S. Sacks, and D. A. Vorp. The effects of aneurysm on the biaxial mechanical behavior of human abdominal aorta. J. Biomech., 39:1324-1334, 2006.

[62] G. A. Holzapfel, M. Stadler, and C. A. J. Schulze-Bauer. A layer-specific three-dimensional model for the simulation of balloon angioplasty using magnetic resonance imaging and mechanical testing. Ann. Biomed. Eng., 30:753-767, 2002. 